\title{
25. PETROGRAPHIC AND CHEMICAL CHARACTERISTICS OF ABYSSAL THOLEIITES FROM DEEP SEA DRILLING PROJECT LEG 63 OFF BAJA CALIFORNIA ${ }^{1}$
}

\author{
Tsugio Shibata, Department of Earth Sciences, Okayama University, Tsushima, Okayama 700, Japan \\ and \\ Stephen E. DeLong and Peter Lyman², Department of Geological Sciences, State University of New York \\ at Albany, Albany, New York
}

\begin{abstract}
Tholeiitic basalts were obtained from basaltic basement ranging in age from 6 to $17 \mathrm{~m} . \mathrm{y}$. on IPOD/DSDP Leg 63 . The main rock types encountered at all sites but 473 are basaltic pillow lavas. Although many of these pillow basalts are highly or moderately altered, fresh glass is usually present. At Site 473, we recovered coarse-grained, massive basalts; no clearly defined pillowed forms were observed. Phenocrysts or microphenocrysts present in the Leg 63 basalts are plagioclase and clinopyroxene at Site 469; olivine, plagioclase, and spinel at Site 470; and olivine, plagioclase, and clinopyroxene at Sites 472 and 473 . Olivines of the basalts from Holes $470 \mathrm{~A}$ and $472\left(\mathrm{Fo}_{85}-88\right)$ are generally more magnesian than those of the Hole 473 basalts $\left(\mathrm{Fo}_{77-81}\right)$. Also, plagioclases of Holes $470 \mathrm{~A}$ and 472 basalts $\left(\mathrm{An}_{70-85}\right)$ are generally more calcic than those of Holes 469 and 473 basalts $\left(\mathrm{An}_{66-72}\right)$.

Geochemical study of the Leg 63 basalts indicates that in all cases they are large-ion-lithophile (LIL) element depleted tholeiites like typical abyssal tholeiites. In particular, they are very similar in composition to those described from the eastern Pacific, although the degree of iron enrichment found in the Leg 63 basalts is not as extensive as in basalts from the Galapagos spreading center. Hence, the geochemical evidence of the Leg 63 basalts is compatible with their formation at a spreading center. Compositional variations in Leg 63 basalts from any single drill hole is small. Major and trace element data indicate that the samples from Holes 469 and 473 are more fractionated in chemical composition than are the samples from Holes $470 \mathrm{~A}$ and 472 ; this compositional variation may be largely ascribed to differences in the extent of shallow-level fractional crystallization of similar parental magma. The Hole 472 samples, however, show a LIL element character distinct from the other Leg 63 samples.
\end{abstract}

\section{INTRODUCTION}

Drilling terminated in basaltic basement at four of the seven sites during IPOD/DSDP Leg 63 off Baja California. In this report, we present petrographic and geochemical data on basalt samples obtained from DSDP Leg 63 Holes 469, 470A, 472, 472A, and 473 (Fig. 1) and discuss their preliminary petrogenetic interpretations. Pillow basalts are the main component of the uppermost basaltic basement at all the sites except 473 , where we recovered coarse-grained, massive basalts that could represent thick-ponded lava flows or intrusives. The oldest sediment that rests upon the basaltic basement at each of these sites ranges in age from 6 to 17 m.y. Because Sites 469, 470, and 472 are located at significant distances from each other on ocean floor of similar age (15-17 m.y.; Fig. 1), the samples obtained at these sites provide information concerning spatial variation in petrological and geochemical parameters along the past spreading center.

Lithology of the basaltic basement recovered during DSDP Leg 63 is summarized in Fig. 2. Details of the geology of the drilling sites and hole summaries are given elsewhere in this volume. We encountered diabasic intrusives at Sites 469 and 471 . Petrography and

\footnotetext{
${ }_{2}^{1}$ Initial Reports of the Deep Sea Drilling Project, Volume 63.

2 Mr. Lyman's present address: Department of Earth and Space Sciences, SUNY at Stony Brook, Stony Brook, New York.
}

geochemistry of these samples are treated separately (Shibata and DeLong, this volume).

\section{PETROGRAPHY}

\section{Hole 469}

The basaltic pillow lavas and basaltic pebbles encountered at Site 469 are gray to dark gray; they become brownish gray along fractures and veinlets and are commonly this color along the glassy chilled zones. Vesicles are not abundant $(<4 \%)$ and are generally small $(<1.5$ $\mathrm{mm}$ ). Veinlets and cracks generally filled with greenish clay minerals and calcite are common. The pillow basalts and pebble-sized basaltic fragments are mostly aphanitic, and no phenocryst phases are visible. Petrographic study indicates that these basalts are mostly aphyric, but some are porphyritic with trace amounts $(\leq 1 \%)$ of plagioclase microphenocrysts or plagioclase plus clinopyroxene microphenocrysts. These microphenocrysts are commonly euhedral and $0.2 \mathrm{~mm}$ to $1.5 \mathrm{~mm}$ in length. In one specimen sampled from Core 43 (Section 3, 73-75 cm), we found olivine microphenocryst pseudomorphs that are now completely made up of talc, smectite, and magnetite, but all other specimens examined under the microscope are entirely devoid of olivine and also Ca-poor pyroxene.

The textures of aphyric basalts and those of the groundmass of plagioclase or plagioclase-clinopyroxene phyric basalts vary from holohyaline to intersertal and locally subvariolitic or variolitic. They are, except for 


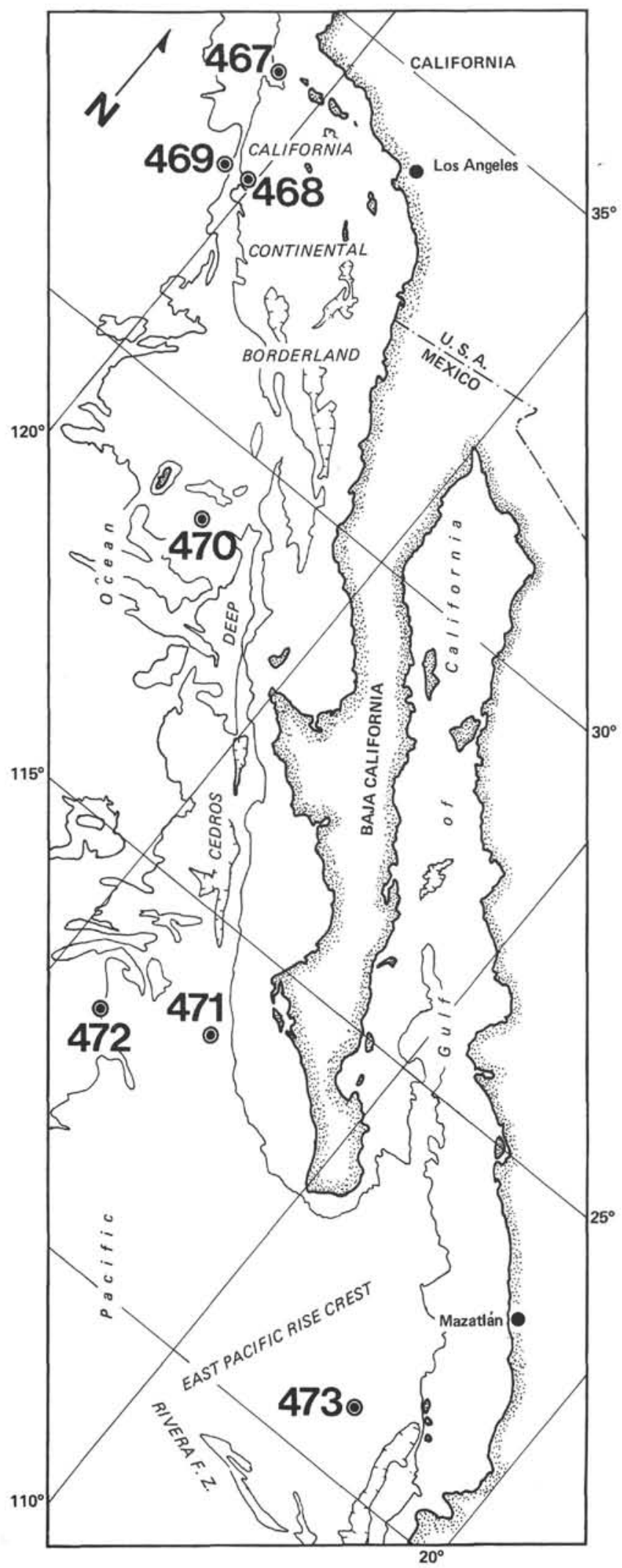

Figure 1. Locations of Leg 63 drill sites. those with holohyaline texture, characterized by skeletal microlites of plagioclase and clinopyroxene with well developed quench morphology.

Mineral analyses are available only for plagioclase microphenocrysts (see the detailed discussion that follows). These microphenocrysts have a compositionally homogeneous, broad core of $\mathrm{An}_{70}$ with a thin veneer of more sodic plagioclase.

The pillow basalts from the uppermost part of the basement at Site 469 are strongly altered and show a clay-rich altered mesostasis. Alteration effects become moderate lower in the recovered basalt sequence where secondary minerals are generally confined to vesicles and cracks.

\section{Hole 470A}

The basaltic pillows obtained from cores at Site 470 are usually characterized by a dark brown, glassy margin $(\simeq 1 \mathrm{~cm})$, which generally grades into a narrow, brownish aphanitic zone ( $2-3 \mathrm{~cm}$ thick) and then into a porphyritic, fine to medium-grained interior with greenish, altered olivine phenocrysts. Petrographic study indicates that these basalts are for the most part porphyritic with plagioclase microphenocrysts or plagioclase plus olivine microphenocrysts $(0.5-1.5 \mathrm{~mm}$ in diameter) set in an intersertal to intergranular groundmass, but the pillow margins and centers are usually characterized by subvariolitic and subophitic texture, respectively. The olivine microphenocrysts are commonly partly or completely replaced by brownish green clay, calcite, and/or serpentine. The microprobe analyses of unaltered olivine microphenocrysts indicate that they show virtually no chemical zoning and range in composition from $\mathrm{Fo}_{88}$ to $\mathrm{Fo}_{85}$. Plagioclase microphenocrysts are euhedral and have a compositionally homogeneous, broad core of $\mathrm{An}_{85-70}$ with a thin veneer of more sodic plagioclase.

The groundmass of the basaltic pillow margins is characterized by skeletal microlites of plagioclase, feathery and dendritic clinopyroxene, skeletal opaque minerals, and equant olivine set in an altered mesostasis. These quenched mineral morphologies become less prominent toward pillow interiors, however, where the groundmass is characterized by a subophitic texture with plagioclase laths embayed in larger, anhedral clinopyroxenes. These clinopyroxenes fall in the compositional ranges of salite and magnesian augite in the conventional pyroxene quadrilateral (Fig. 3).

Basalts obtained from Holes 470 and 470A are mostly olivine-plagioclase phyric, and no clinopyroxene is observed as a phenocryst phase. Moreover, olivine is commonly found as one of the groundmass minerals. These petrographic observations suggest that basalts of Holes 470 and $470 \mathrm{~A}$ are on the olivine-plagioclase cotectic and, compared to pyroxene-plagioclase phyric basalts obtained at Site 469 , are more primitive.

\section{Hole 472}

The basaltic pillow lavas and basaltic fragments are gray to dark gray and become brownish gray along the 


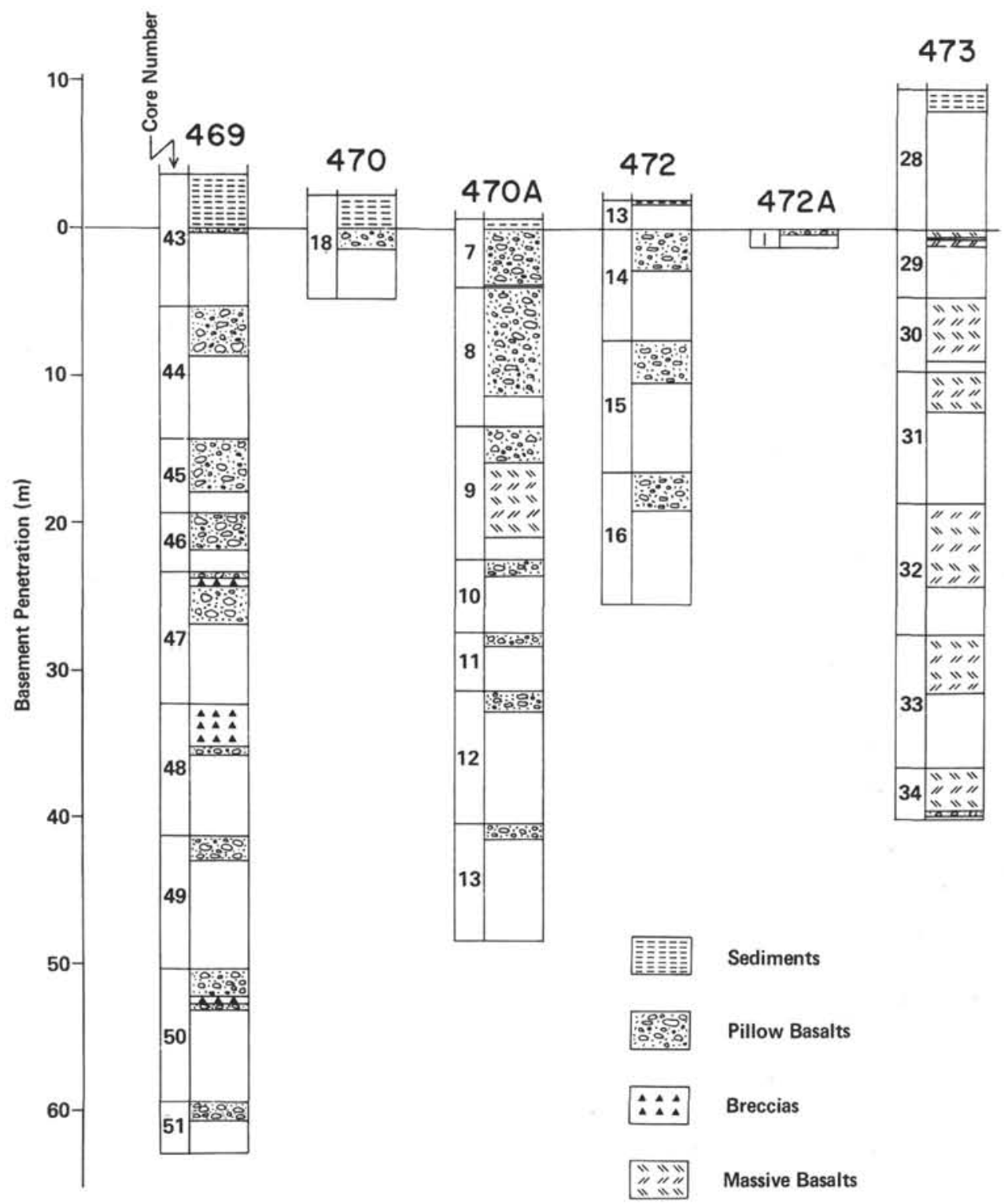

Figure 2. Lithologic summary of DSDP Leg 63 basaltic basement. (Depths are in meters from the sediment/basaltic basement interface.)

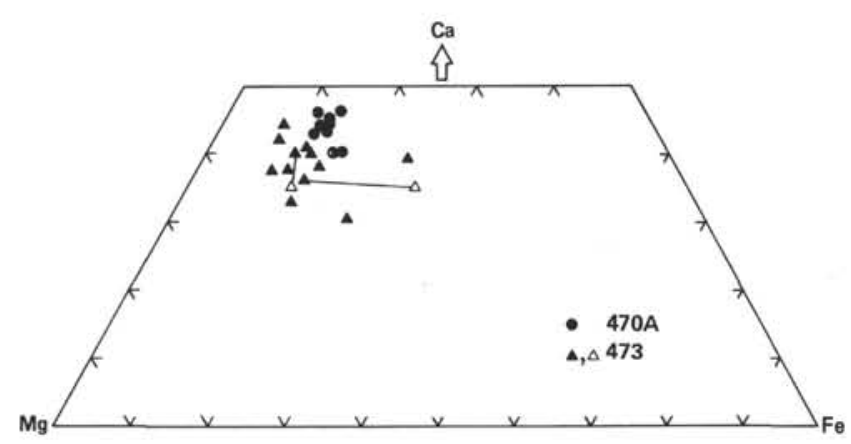

Figure 3. Pyroxene compositions in atomic $\mathrm{Ca}-\mathrm{Mg}$ - $\mathrm{Fe}$ proportions. (Filled circles, Hole 470A; triangles, Hole 473. Compositions of core $[\mathbf{\Delta}]$-margin $[\Delta]$ pairs are connected by tie-lines.) glassy chilled margins. These basalts are vesicular, but vesicles are not abundant $(<2-3 \%)$, are generally small in size $(<0.5 \mathrm{~mm})$, and are commonly filled with calcite and clay minerals. The pillow basalts recovered at Site 472 are more fragmented than those at Site 470 , but the general pillow structures are recognizable. The pillow basalts and pebble-size basaltic fragments are mostly massive and aphanitic. Petrographic study indicates that the textures of these basalts vary from aphyric to porphyritic and glomeroporphyritic with plagioclase microphenocrysts occasionally accompanied by olivine microphenocrysts. These microphenocrysts are generally euhedral and $0.2 \mathrm{~mm}$ to $1 \mathrm{~mm}$ long. The olivine microphenocrysts show virtually no chemical zoning and are $\mathrm{Fo}_{85-87}$. The plagioclase microphenocrysts are normally zoned and show a composition range of $\mathrm{An}_{77-82}$. 
The pillow interiors usually show an intergranular texture with minute mesostasis between plagioclase laths and equant clinopyroxenes. The groundmass textures of specimens sampled from pillow margins vary from holohyaline to subvariolitic, and locally to hyalopilitic, and are characterized by skeletal microlites of plagioclase and feathery clinopyroxene with well developed quench morphology. The glassy fragments found in the hyaloclastic breccias are partly devitrified, but are mostly fresh and clear brown. They are angular and range in size from $1 \mathrm{~mm}$ to $50 \mathrm{~mm}$. Euhedral plagioclase occurs commonly as a microphenocryst phase in those glassy fragments, but it is not abundant $(<2 \%)$.

\section{Hole 473}

The main rock type encountered at Site 473 is coarsegrained, massive basalt. Although aphanitic, finegrained basalts occur at the top, bottom, and an intermediate horizon (Core 32) of Hole 473, no clearly identified basaltic pillow lava is observed. Because of poor recovery at Site 473 , the exact contact relation between the basaltic basement and the overlying sediments is unknown. Also, intercalated soft materials (presumably sediments) were apparently encountered at the depth interval of Core 32 , but none of these was recovered. For these reasons, the mode of occurrence of these massive basalts could be either intrusive or extrusive. Chemical evidence presented later suggests, however, that it is unlikely that these massive basalt units represent a single sill, because the basalts are chemically more differentiated downward in the hole, a trend opposite to that expected from simple fractional crystallization. Recent observations of DSDP deep basement holes (Hall and Robinson, 1979) and direct observations from submersibles (e.g., Ballard et al., 1979 ) indicate that sheet flows represent a relatively common mode of occurrence of extrusive submarine basalts. Although the evidence is inconclusive, the massive basalt units observed in Hole 473 may represent submarine sheet flows or ponded lava flows.

Petrographic analysis of basalt from the top of the basaltic basement at Site $\mathbf{4 7 3}$ indicates that the specimen is glomeroporphyritic with plagioclase and clinopyroxene phenocrysts set in an intersertal to subvariolitic groundmass. Olivine pseudomorphs that are now entirely composed of calcite and clay are also found together with plagioclase and clinopyroxene phenocrysts. The groundmass is composed mainly of plagioclase microlites, equant clinopyroxene, and opaque minerals set in a clay-rich altered mesostasis.

The massive, coarse-grained basalts are petrographically quite similar throughout the hole, and no significant mineralogical variation is observed. The massive basalts are composed mainly of plagioclase and clinopyroxene with subordinate amounts of olivine and opaque minerals. They are extensively altered with abundant greenish clay occupying the interstices between subhedral plagioclase laths and anhedral clinopyroxenes. Pseudomorphs that were probably formed after olivines, but are composed of clay and calcite, occur occasionally. The textures of these massive basalts vary from intersertal to subophitic, and plagioclases and clinopyroxenes generally range in grain size from $1.0 \mathrm{~mm}$ to 1.5 $\mathrm{mm}$. The plagioclases are normally zoned, showing compositional ranges generally from $\mathrm{An}_{72-66}$ at cores to $\mathrm{An}_{67-60}$ at rims. And the clinopyroxenes are strongly zoned (see Fig. 3).

\section{MINERAL CHEMISTRY}

\section{METHODS}

Selected mineral analyses for the Leg 63 basalts are listed in Tables $1,2,3$, and 4 . These mineral compositions were obtained at Hiroshima University using a JEOL X-ray microprobe analyzer (JXA-5A) operated at an accelerating voltage of $15 \mathrm{kV}, 0.01$ to 0.02 $\mu \mathrm{A}$ sample current, and 2 to $3 \mu \mathrm{m}$ spot size. The correction procedure was that of Bence and Albee (1968) with the alpha factors of Nakamura and Kushiro (1970).

\section{Olivine}

Olivine ranging from $\mathrm{Fo}_{77}$ to $\mathrm{Fo}_{88}$ is a conspicuous phenocryst or microphenocryst in the Leg 63 basalts. Compositional variation of olivine within a single grain and a given thin section or even in a given hole is small. Olivine compositions are generally more $\mathrm{Mg}$-rich in the basalts of Holes 470A and 472 than in those of Hole 473 (Table 1). As will be shown later, this is broadly consistent with the $\mathrm{FeO}^{*}$ (total iron as $\mathrm{FeO}$ ) $/ \mathrm{MgO}$ variation of the Leg 63 basalt; i.e., the $\mathrm{FeO}^{*} / \mathrm{MgO}$ ratios of basalts from Holes $470 \mathrm{~A}$ and 472 tend to be lower than those from Hole 473. Two pairs of co-existing olivine and basalt glass analyses (Samples 470A-8-5, 74-80 cm and 472-14-1, 47-53 cm) are available (Tables 1 and 9). The distribution of $\mathrm{Fe}$ and $\mathrm{Mg}$ between the coexisting olivine and basalt glasses gives $K_{D}$ values of 0.28 for the Hole $470 \mathrm{~A}$ pair and 0.33 for the Hole 472 pair. These values conform well to the observed $K_{D}$ values $(0.30$ 0.33 ) of Roeder and Emslie (1970) for equilibrium of olivine and basaltic liquid, indicating that these olivines are probably in equilibrium with surrounding basaltic glass.

\section{Spinel}

Spinels found in the basalts of Hole $470 \mathrm{~A}$ are magnesiochromites, ranging in $100 \times \mathrm{Mg} / \mathrm{Mg}+\mathrm{Fe}^{2+}$ from 69 to 64 and in $100 \times \mathrm{Cr} / \mathrm{Cr}+\mathrm{Al}$ from 56 to 46 (Table 2). They are generally similar in composition to magnesiochromites observed commonly in abyssal tholeiites (Sigurdsson and Schilling, 1976); and $\mathrm{Mg}-\mathrm{Fe}^{2+}$ distribution relations of spinel and olivine coexisting in a given thin section are comparable to those described by Sigurdsson and Schilling for the Mid-Atlantic Ridge spinel-olivine pairs. Spinels are observed only in the basalts of Hole 470A. This fact may suggest that these basalts are relatively less fractionated than the other Leg 63 basalts, because spinel frequently ceases crystallizing at an early stage during evolution of common basalt magmas (e.g., Irvine, 1967; Sigurdsson and Schilling, 1976; Shibata, Thompson, and Frey, 1979).

\section{Pyroxene}

Pyroxene analyses are available only for the basalts of Holes 470A and 473, and all pyroxene analyses listed 
Table 1. Representative microprobe analyses of olivine.

\begin{tabular}{|c|c|c|c|c|c|c|c|c|c|c|c|c|c|c|c|c|c|}
\hline Analysis No. ${ }^{a}$ & 1 & 2 & 3 & 4 & 5 & 6 & 7 & 8 & 9 & 10 & 11 & 12 & 13 & 14 & 15 & 16 & 17 \\
\hline $\mathrm{SiO}_{2}$ & 40.09 & 40.24 & 39.75 & 40.19 & 39.63 & 39.61 & 38.87 & 39.52 & 39.75 & 40.05 & 40.12 & 40.16 & 37.52 & 37.64 & 36.23 & 37.16 & 36.44 \\
\hline $\mathrm{TiO}_{2}^{2}$ & 0.04 & 0.04 & 0.03 & 0.04 & 0.03 & 0.03 & 0.03 & 0.03 & 0.02 & 0.02 & 0.04 & 0.03 & 0.04 & 0.05 & 0.05 & 0.05 & 0.06 \\
\hline $\mathrm{Al}_{2} \mathrm{O}_{3}$ & 0.09 & 0.10 & 0.09 & 0.09 & 0.08 & 0.08 & 0.05 & 0.08 & 0.07 & 0.33 & 0.06 & 0.06 & 0.05 & 0.06 & 0.07 & 0.07 & 0.32 \\
\hline $\mathrm{FeO} *$ & 11.79 & 11.79 & 12.42 & 11.30 & 12.86 & 13.02 & 14.51 & 12.93 & 12.92 & 13.70 & 13.52 & 13.44 & 19.06 & 18.74 & 21.07 & 19.46 & 18.34 \\
\hline $\mathrm{MnO}$ & 0.24 & 0.24 & 0.24 & 0.23 & 0.22 & 0.25 & 0.27 & 0.29 & 0.25 & 0.26 & 0.29 & 0.31 & 0.34 & 0.36 & 0.42 & 0.38 & 0.36 \\
\hline $\mathrm{MgO}$ & 48.06 & 47.94 & 47.51 & 48.17 & 46.57 & 46.39 & 45.64 & 46.42 & 45.22 & 44.99 & 44.47 & 44.80 & 42.23 & 42.48 & 39.87 & 41.99 & 42.58 \\
\hline $\mathrm{CaO}$ & 0.33 & 0.35 & 0.37 & 0.33 & 0.34 & 0.34 & 0.38 & 0.33 & 0.32 & 0.37 & 0.38 & 0.36 & 0.35 & 0.34 & 0.32 & 0.34 & 0.33 \\
\hline Total & 100.64 & 100.70 & 100.41 & 100.35 & 99.73 & 99.71 & 99.75 & 99.60 & 98.55 & 99.72 & 98.88 & 99.16 & 99.59 & 99.67 & 98.03 & 99.45 & 98.43 \\
\hline \multicolumn{18}{|c|}{ Formula basis $=4$ oxygens } \\
\hline $\mathrm{Si}$ & 0.987 & 0.990 & 0.984 & 0.990 & 0.990 & 0.990 & 0.980 & 0.989 & 1.004 & 1.002 & 1.012 & 1.009 & 0.970 & 0.971 & 0.964 & 0.965 & 0.953 \\
\hline Al & 0.003 & 0.003 & 0.003 & 0.003 & 0.002 & 0.002 & 0.001 & 0.002 & 0.002 & 0.010 & 0.002 & 0.002 & 0.002 & 0.002 & 0.002 & 0.002 & 0.010 \\
\hline $\mathrm{Ti}$ & 0.001 & 0.001 & 0.001 & 0.001 & 0.001 & 0.001 & 0.001 & 0.001 & 0.000 & 0.000 & 0.001 & 0.001 & 0.001 & 0.001 & 0.001 & 0.001 & 0.001 \\
\hline $\mathrm{Mg}$ & 1.764 & 1.758 & 1.754 & 1.768 & 1.734 & 1.729 & 1.715 & 1.732 & 1.702 & 1.678 & 1.671 & 1.678 & 1.627 & 1.633 & 1.580 & 1.625 & 1.659 \\
\hline $\mathrm{Fe}^{2}+$ & 0.243 & 0.243 & 0.257 & 0.233 & 0.269 & 0.272 & 0.306 & 0.271 & 0.273 & 0.287 & 0.285 & 0.283 & 0.412 & 0.404 & 0.469 & 0.423 & 0.401 \\
\hline $\mathrm{Mn}$ & 0.005 & 0.005 & 0.005 & 0.005 & 0.005 & 0.005 & 0.006 & 0.006 & 0.005 & 0.006 & 0.006 & 0.007 & 0.007 & 0.008 & 0.009 & 0.008 & 0.008 \\
\hline $\mathrm{Ca}$ & 0.009 & 0.009 & 0.010 & 0.009 & 0.009 & 0.009 & 0.010 & 0.009 & 0.009 & 0.010 & 0.010 & 0.010 & 0.010 & 0.009 & 0.009 & 0.009 & 0.009 \\
\hline Total & 3.012 & 3.009 & 3.014 & 3.009 & 3.010 & 3.008 & 3.019 & 3.010 & 2.995 & 2.993 & 2.987 & 2.990 & 3.029 & 3.028 & 3.034 & 3.033 & 3.041 \\
\hline Fo mole $\%$ & 87.9 & 87.9 & 87.2 & 88.4 & 86.6 & 86.4 & 84.9 & 86.5 & 86.2 & 85.4 & 85.4 & 85.6 & 79.8 & 80.2 & 77.1 & 79.4 & 80.5 \\
\hline
\end{tabular}

Note: $\mathrm{FeO}^{*}=$ total iron as $\mathrm{FeO}$.

a Analysis numbers designate the following samples: 1: Phenocryst (core), 470A-7-2, 119-126 cm. 2: Phenocryst (rim), the same olivine grain as Analysis No. 1. 3: MicrophenQcryst (core), 470A-7-2, 119-126 cm. 4: Phenocryst (core), 470A-7-2, 119-126 cm. 5: Phenocryst (core), 470A-8-5, 74-80 cm. 6: Phenocryst (rim), the same olivine grain as Analysis No. 5. 7: Microphenocryst, 470A-9-5, 74-81 cm. 8: Phenocryst (core), 472-14-1, 47-53 cm. 9: Phenocryst (rim), the same olivine grain as Analysis No. 8. 10: Microphenocryst (core), 472-14-2, $32-38 \mathrm{~cm}$. 11: Microphenocryst (rim), the same olivine grain as Analysis No. 10. 12: Microphenocryst, 472-14-2, 32-38 cm. 13: 473-33-3, 92-97 cm. 14: 473-33-3, 92-97 cm. 15: 473-34$1,25-31 \mathrm{~cm}$. 16: 473-34-1, 25-31 cm. 17: 473-34-1, 25-31 cm.

Table 2. Microprobe analyses of spinel.

\begin{tabular}{lrrrr}
\hline Analysis No. $^{\mathrm{a}}$ & \multicolumn{1}{c}{1} & \multicolumn{1}{c}{2} & \multicolumn{1}{c}{3} & \multicolumn{1}{c}{4} \\
\hline $\mathrm{SiO}_{2}$ & 0.12 & 0.15 & 0.14 & 0.10 \\
$\mathrm{TiO}_{2}$ & 0.60 & 0.58 & 0.56 & 0.68 \\
$\mathrm{Al}_{2} \mathrm{O}_{3}$ & 29.39 & 29.29 & 28.88 & 23.06 \\
$\mathrm{Cr}_{2} \mathrm{O}_{3}$ & 36.87 & 37.55 & 38.09 & 43.32 \\
$\mathrm{FeO}^{*}$ & 17.78 & 17.63 & 17.43 & 19.21 \\
$\mathrm{MnO}$ & 0.23 & 0.29 & 0.27 & 0.33 \\
$\mathrm{MgO}$ & 16.09 & 15.93 & 15.86 & 14.56 \\
$\mathrm{CaO}$ & 0.04 & 0.07 & 0.04 & 0.02 \\
$\mathrm{Total}$ & 101.12 & 101.49 & 101.27 & 101.28 \\
Formula basis & 4 oxygens & & & \\
$\mathrm{Si}$ & 0.003 & 0.004 & 0.004 & 0.003 \\
$\mathrm{Al}$ & 1.004 & 1.000 & 0.989 & 0.814 \\
$\mathrm{Ti}$ & 0.013 & 0.013 & 0.012 & 0.015 \\
$\mathrm{Fe}$ & 0.117 & 0.107 & 0.103 & 0.122 \\
$\mathrm{Cr}$ & 0.845 & 0.860 & 0.875 & 1.026 \\
$\mathrm{Mg}$ & 0.695 & 0.688 & 0.687 & 0.650 \\
$\mathrm{Fe}$ & 0.314 & 0.320 & 0.321 & 0.359 \\
$\mathrm{Mn}$ & 0.006 & 0.007 & 0.007 & 0.008 \\
$\mathrm{Ca}$ & 0.001 & 0.002 & 0.001 & 0.001 \\
$\mathrm{Mg} / \mathrm{Mg}+\mathrm{Fe}^{2+}$ & 68.9 & 68.3 & 68.2 & 64.4 \\
\hline
\end{tabular}

Note: $\mathrm{FeO}^{*}=$ total iron as $\mathrm{FeO}$. Ferric and ferrous iron are calculated from total iron and structural formula (Finger, 1972).

a Analysis numbers designate the following samples: 1, 2, 3: Brown spinels $(\simeq 0.1 \mathrm{~mm}$ across) enclosed in plagioclase phenocryst, $470 \mathrm{~A}-7-2,119-126 \mathrm{~cm}$. 4: Brown spinel $(0.2 \mathrm{~mm}$ across) enclosed in olivine phenocryst (Analysis No. 4 in Table 1), $470 \mathrm{~A}-7-2,119-126 \mathrm{~cm}$.

in Table 3 are plotted in the pyroxene quadrilateral (Fig. 3). No Ca-poor pyroxene was observed in any of the specimens analyzed. The pyroxenes are mostly augites, and there is a distinct compositional difference between the pyroxenes from Holes $470 \mathrm{~A}$ and 473 , i.e., the pyroxenes from Hole 470A tend to be more calcic and have higher $\mathrm{FeO}^{*} / \mathrm{MgO}$ ratios than those from Hole 473. The olivines with which these pyroxenes coexist are more $\mathrm{Mg}$-rich in the basalts from Hole 470A than those from Hole 473, and the $\mathrm{FeO} * / \mathrm{MgO}$ ratios of the host basalts from which these pyroxene analyses were obtained are higher in the Hole 473 basalts than in the Hole $470 \mathrm{~A}$ basalts.

\section{Plagioclase}

The anorthite contents of plagioclase observed as phenocrysts and microphenocrysts of the Leg 63 basalts are quite variable, ranging from $\mathrm{An}_{85}$ to $\mathrm{An}_{60}$ (Table 4). Microprobe analyses indicate that the plagioclase phenocrysts and microphenocrysts found in the basalts from Holes $470 \mathrm{~A}$ and 472 are generally more calcic than those found in the basalts from Holes 469 and 473, a tendency in accord with the olivine compositional variation discussed previously.

$\mathrm{Fe}$ and $\mathrm{Mg}$ substitute as a $\mathrm{Ca}(\mathrm{Fe}, \mathrm{Mg}) \mathrm{Si}_{3} \mathrm{O}_{8}$ component in basaltic plagioclase (Bryan, 1974; Longhi, 1976; Longhi et al., 1976), and the abundances of $\mathrm{Fe}$ and $\mathrm{Mg}$ generally decrease from groundmass to microphenocryst plagioclases (Shibata, DeLong, and Walker, 1979). The plagioclase analyses from the Leg 63 basalts are plotted in the $(\mathrm{Fe}, \mathrm{Mg})_{2} \mathrm{Si}_{3} \mathrm{O}_{8}-\mathrm{Ca}_{2} \mathrm{Si}_{3} \mathrm{O}_{8}-\mathrm{Al}_{4} \mathrm{SiO}_{8}$ diagram (Fig. 4), which is the excess component plane of the system ( $\mathrm{Fe}, \mathrm{Mg})_{2} \mathrm{Si}_{3} \mathrm{O}_{8}-\mathrm{Ca}_{4} \mathrm{Si}_{2} \mathrm{O}_{8}-\mathrm{Al}_{8} \mathrm{Si}_{-2} \mathrm{O}_{8}$ - $\mathrm{Si}_{4} \mathrm{O}_{8}$ (Longhi, 1976; Longhi et al., 1976). This figure indicates that, as expected, $\mathrm{Fe}$ and $\mathrm{Mg}$ substitute mainly as a $\mathrm{Ca}(\mathrm{Fe}, \mathrm{Mg}) \mathrm{Si}_{3} \mathrm{O}_{8}$ component in the Leg 63 plagioclases. The extent of this substitution is similar to that in the phenocryst plagioclases but not as much as in the groundmass plagioclases of the Oceanographer fracture zone basalts (Shibata, DeLong, and Walker, 1979).

\section{BULK ROCK COMPOSITION}

\section{METHODS}

Bulk rock major element data were obtained, except for ferrous iron and $\mathrm{H}_{2} \mathrm{O}$, by electron microprobe analyses of fused glass beads prepared from rock powders (Suzuki et al., 1977). Approximately 500 $\mathrm{mg}$ to $800 \mathrm{mg}$ splits of $150 \mathrm{~g}$ rock powders were pressed at load pressures between 2000 and $3000 \mathrm{~kg} / \mathrm{cm}^{2}$ to make sample tablets 10 $\mathrm{mm}$ in diameter and $3 \mathrm{~mm}$ to $5 \mathrm{~mm}$ thick. These tablets were then placed in a plasma torch and fused in an argon atmosphere. 
Table 3. Microprobe analyses of clinopyroxene.

\begin{tabular}{|c|c|c|c|c|c|c|c|c|c|c|c|c|c|c|c|c|c|c|c|c|c|c|c|}
\hline nalysis No.a & 1 & 2 & 3 & 4 & 5 & 6 & 7 & 8 & 9 & 10 & 11 & 12 & 13 & 14 & 15 & 16 & 17 & 18 & 19 & 20 & 21 & 22 & 23 \\
\hline $\mathrm{SiO}_{2}$ & 48.20 & 50.31 & 48.72 & 49.25 & 48.37 & 47.44 & 49.08 & 50.28 & 49.15 & 50.33 & .16 & 51.53 & 51.40 & 49.77 & 47.90 & 52.61 & 52.13 & 48.97 & 50.62 & 49.62 & 50.32 & 50.10 & 51.64 \\
\hline & & & & & .22 & 16 & 1.48 & 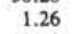 & 1.18 & & .56 & 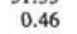 & & & 1.58 & (1) & 0.92 & 0.85 & 0.81 & 145 & 1.28 & 0.85 & 0.62 \\
\hline & 3.83 & & 4.93 & & 3.41 & 5.26 & 4.57 & 3.49 & 2.76 & 3. & 3.02 & 2. & 1.56 & 0.97 & 2.26 & 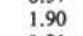 & 1.59 & 3.32 & 1.38 & 4.57 & 4.05 & 56 & 1.60 \\
\hline & 6.92 & & 8.26 & & $\begin{array}{r}3.471 \\
10.49\end{array}$ & 8.71 & 7.65 & 7.52 & 10.81 & 4. & 5.14 & 6. & 9.10 & 17.59 & 15.50 & 1 & 7.54 & & 14.26 & 8.01 & 9.86 & 24 & 8.70 \\
\hline & 0.23 & & 0.28 & & 0.36 & & 0.27 & 0.26 & 0.38 & & 0.20 & & 0.33 & & 0.49 & & 0.30 & 0.28 & 0.48 & 0.26 & 0.35 & 0.25 & 0.29 \\
\hline & & & & & & & S & & & & & & . & & & & & & 15.27 & $i$ & 18.19 & 19.32 & 17.19 \\
\hline & & & 40 & & 0.32 & & 0.00 & 0.00 & 0.00 & 0.30 & 0.30 & 0.2 & 0.23 & 0. & 0.44 & 0.18 & 0.21 & 0.24 & 0.21 & 0.28 & 0.27 & 0.28 & 0.19 \\
\hline Total & 97.23 & 99.77 & 99.59 & 99.73 & 98.49 & 99.26 & 99.58 & 99.35 & 98.91 & 97.79 & 98.54 & 98.73 & 98.36 & 98.58 & 97.68 & 100.65 & 100.25 & 98.03 & 99.62 & 99.51 & 100.41 & 98.31 & 98.34 \\
\hline \multicolumn{24}{|c|}{ ormula basis $=6$ oxygens } \\
\hline $\mathrm{Si}$ & 1.846 & 1.881 & 1.82 & 1.8 & 1.84 & 1.7 & 1.8 & 1.8 & 1.8 & 1.85 & 1.9 & 1.9 & 1.9 & 1.5 & 1.8 & 1 . & 1.9 & 1.8 & & 1.84 & 1.8 & 1.879 & 1.935 \\
\hline $\mathrm{T}$ & & 0. & & & & & & & & & & & & & & & & & & & & & 17 \\
\hline & & & & & & & & & & & & & & & & & & & & & & & 11 \\
\hline & & & & & & & & & & & & & & & & & & & & & & & 0.273 \\
\hline M & & & & & & & & & & & & & & & & & & & & & & & \\
\hline 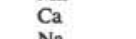 & & & & & & & & & & & & & & & & & & & & & & & \\
\hline $\mathrm{N}$ & & & & & & & & & 0. & 0.8 & $0 . c$ & 0. & 0.0 & 0. & & & & 0.018 & 0.0 & 20 & 0.019 & 0.020 & 0.014 \\
\hline Total & 4.030 & 4.030 & 4.025 & 4.049 & 4.052 & 4.053 & 4.025 & 4.012 & 4.033 & 4.039 & 4.027 & 4.035 & 4.024 & 4.019 & 4.033 & 4.024 & 4.030 & 4.053 & 4.037 & 4.021 & 4.025 & 4.026 & 4.020 \\
\hline $\mathrm{C}$ & & & & & & & & & & & & & & & & & & & & & 377 & & 35.0 \\
\hline & & & & & & & & & & & & & & & & & & & & & & & \\
\hline $\mathbf{F}$ & 11.5 & 13.2 & 3.7 & 13.7 & 9 & 14.2 & 3 & 12.1 & 17.3 & .6 & 8.2 & .9 & 14.5 & 29.3 & 26.2 & 14.3 & 11.6 & 2. & 2.5 & 3 . & 15.9 & 11.7 & 3.8 \\
\hline
\end{tabular}

Note: $\mathrm{FeO}^{*}=$ total iron as FeO.
a Analysis nunbers designate the following samples: 1: Subhedral clinopyroxene (core), 470A-9-3, 99-104 cm. 2: Subhedral clinopyroxene (rim), the same pyroxene grain as Analysis No. 1. 3: 470A-9-3, 99-104 cm. 4, 5, 6: Groundmass pyroxene, 470A-9-5, 74-81 cm. 7, 8, 9: Acicular groundmass pyroxene, all analyses are obtained from a single grain, 470A-13-1, 28-33 cm. 10: Microphenocryst, 473-29-1, 70-75 cm. 11: Microphenocryst, 473-29-1, 70-75 cm. 12:

Microphenocryst (core), 473-29-1, 70-75 cm. 13: (core), 473-30-2, 114-120 cm. 14: (rim), the same pyroxene grain as Analysis No. 13. 15: (rim), 473-30-2, 114-120 cm. 16: (core), 473-33-3, 92-97 cm. 17, 18: (core), 473-34-1, 25-31 cm. 19:
(core), 473-34-1, 25-31 cm. 20, 21: 473-34-3, 17-23 cm. 22: (core), 473-34-3, 26-34 cm. 23: (rim), the same grain as Analysis No. 22.

Table 4. Representative microprobe analyses of plagioclase.

\begin{tabular}{|c|c|c|c|c|c|c|c|c|c|c|c|c|c|c|c|c|c|c|c|c|c|}
\hline Analysis $\mathrm{No}^{\mathrm{a}}$ & 1 & 2 & 3 & 4 & 5 & 6 & 7 & 8 & 9 & 10 & 11 & 12 & 13 & 14 & 15 & 16 & 17 & 18 & 19 & 20 & 21 \\
\hline $\mathrm{SiO}_{2}$ & 50.50 & 50.86 & $\begin{array}{r}50.60 \\
0.08\end{array}$ & 50.77 & $\begin{array}{r}46.65 \\
0.06\end{array}$ & $\begin{array}{l}46.25 \\
0.05\end{array}$ & 51.03 & 52.34 & 48.54 & 47.59 & 46.11 & 48.68 & 46.84 & 48.11 & 51.20 & 50.28 & 51.84 & 50.85 & 50.96 & 51.25 & 53.15 \\
\hline $\begin{array}{l}\mathrm{TiO}_{2} \\
\mathrm{Al}_{2} \mathrm{O}_{3}\end{array}$ & $\begin{array}{l}0.07 \\
30.62\end{array}$ & $\begin{array}{r}0.07 \\
30.52\end{array}$ & $\begin{array}{r}0.08 \\
31.03\end{array}$ & $\begin{array}{l}0.08 \\
30.89\end{array}$ & $\begin{array}{l}0.06 \\
32.25\end{array}$ & $\begin{array}{r}0.05 \\
33.41\end{array}$ & $\begin{array}{r}0.07 \\
30.28\end{array}$ & 0.05 & 0.06 & 0.07 & 0.05 & 0.06 & 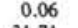 & 0.05 & & 0 & & 10 & 0.08 & & 0.13 \\
\hline $\begin{array}{l}\mathrm{Al}_{2} \mathrm{O}^{3} \\
\mathrm{FeO}{ }^{3}\end{array}$ & $\begin{array}{r}30.62 \\
0.53\end{array}$ & $\begin{array}{l}30.52 \\
0.57\end{array}$ & $\begin{array}{r}31.03 \\
0.52\end{array}$ & $\begin{array}{r}30.89 \\
0.56\end{array}$ & $\begin{array}{l}32.25 \\
0.38\end{array}$ & $\begin{aligned} \begin{array}{r}33.41 \\
0.43\end{array} & \end{aligned}$ & $\begin{array}{l}30.28 \\
0.45\end{array}$ & $\begin{aligned} 29.31 \\
0.61\end{aligned}$ & $\begin{array}{l}31.37 \\
0.58\end{array}$ & $\begin{array}{l}31.54 \\
0.52\end{array}$ & $\begin{array}{l}32.08 \\
0.39\end{array}$ & 31.72 & 31.71 & 31.28 & 29.97 & 31.20 & 30.05 & 29.44 & 30.08 & 29.56 & 28.41 \\
\hline $\mathrm{MnO}$ & 0.04 & 0.03 & 0.02 & 0.04 & 0.04 & 0.02 & 0.03 & 0.01 & 0.05 & 0.03 & 0.03 & 0.02 & 0.04 & 0.02 & 0.01 & $\begin{array}{l}0.50 \\
0.02\end{array}$ & $\begin{array}{l}0.43 \\
0.05\end{array}$ & 0.00 & & $\begin{array}{l}0.56 \\
0.01\end{array}$ & $\begin{array}{l}0.63 \\
0.03\end{array}$ \\
\hline $\mathrm{MgO}$ & 0.24 & 0.24 & 0.22 & 0.23 & 0.23 & 0.21 & 0.26 & 0.38 & 0.20 & 0.23 & 0.24 & 0.24 & 0.18 & 0.19 & 0.26 & 0.25 & 0.27 & 0.21 & 0.24 & 0.20 & 0.23 \\
\hline $\mathrm{CaO}$ & 14.72 & 14.93 & 15.12 & 15.11 & 17.84 & 17.60 & 15.00 & 14.26 & 16.26 & 16.68 & 17.37 & 16.56 & 17.91 & 17.26 & 14.04 & 14.92 & 14.14 & 14.12 & & 14.78 & 13.43 \\
\hline $\mathrm{Na}_{2} \mathrm{O}$ & & & & & & & & & & & & & & & & & & & & & 3.93 \\
\hline $\mathrm{K}_{2} \mathrm{O}$ & 0.05 & 0.04 & 0.06 & 0.05 & 0.04 & 0.04 & 0.05 & 0.06 & 0.04 & 0.05 & 0.05 & 0.06 & 0.05 & 0.07 & 0.05 & 0.04 & 0.05 & 0.06 & 0.06 & 0.06 & 0.07 \\
\hline Total & 100.24 & 100.78 & 101.07 & 101.04 & 99.18 & 100.06 & 100.66 & 100.84 & 99.52 & 99.05 & 98.35 & 100.20 & 99.81 & 100.24 & 99.86 & 100.48 & 100.65 & 99.08 & 99.93 & 99.73 & 100.01 \\
\hline \multicolumn{22}{|c|}{ Formula basis $=8$ oxygens } \\
\hline Si & 305 & 2.311 & & 2.301 & & & 2.3. & 2. & 2.2 & 2.21 & 2.1 & 2.2 & 2.1 & 2.2 & 2.3 & 2.289 & 2.349 & 2. & vit & 18 & 4 \\
\hline $\mathrm{Al}$ & & & & & & & & & & & & & & & & & & & & & .524 \\
\hline M & & & & & & & & & & & & & & & & & & & 003 & 0.002 & .004 \\
\hline $\begin{array}{l}\mathrm{Mg}_{\mathrm{Fe}^{2}+} \\
\text { - }\end{array}$ & & & & & $\begin{array}{l}0 \\
0\end{array}$ & $\begin{array}{l}0.014 \\
0.017\end{array}$ & 0.0 & & 0.014 & $\begin{array}{l}0.016 \\
0.020\end{array}$ & 0.0 & $\begin{array}{l}0.016 \\
0.015\end{array}$ & 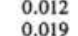 & $\begin{array}{l}0.013 \\
0.021\end{array}$ & 0.018 & $\begin{array}{l}0.017 \\
0.019\end{array}-3$ & $\begin{array}{l}0.018 \\
0.017\end{array}$ & 0.015 & .016 & 0.014 & 2.016 \\
\hline $\mathrm{Mn}$ & & & & & & & & & & & & & 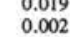 & & $0.6(2-12-3$ & & & & 002 & $\begin{array}{l}0.022 \\
0.001\end{array}$ & $\begin{array}{l}0.001 \\
0.001\end{array}$ \\
\hline $\mathrm{ca}$ & & & & & & & & & & & & & & & & & & & & 0.726 & 0.654 \\
\hline $\mathrm{Na}$ & & & & & & & & & & & & & & & 0. & 0.2 & & 0. & 0.323 & 0.288 & 0.347 \\
\hline $\mathrm{K}$ & 0.003 & 0.00 & 0.00 & 0.00 & 0.002 & 0.002 & 0.003 & 0.003 & 0.003 & 0.003 & 0.003 & 0.004 & 0.00 & 0.004 & 0.00 & 0.00 & 0.003 & 0.004 & 0.004 & 0.004 & 0.004 \\
\hline Total & 5.022 & 5.025 & 5.028 & 5.021 & 5.020 & 5.046 & 5.022 & 5.015 & 5.015 & 5.027 & 5.039 & 5.020 & 5.069 & 5.054 & 5.019 & 5.016 & 5.010 & 5.019 & 5.019 & 5.001 & 4.992 \\
\hline An mole $\%$ & 69.9 & 70.0 & 70.7 & 71.4 & 85.2 & 82.4 & 70.2 & 67.2 & 78.5 & 79.5 & 82.3 & 78.4 & 79.4 & 77.6 & 67.2 & 71.9 & 67.6 & 67.6 & 68.1 & 71.3 & 65.1 \\
\hline
\end{tabular}

Note: $\mathrm{FeO}^{*}=$ total iron as $\mathrm{FeO}$.

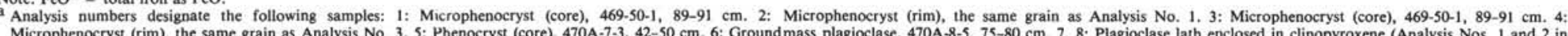
Table 3), 470A-9-3, 99-104 cm. 9: Microphenocryst (rim), 470A-11-1, 68-74 cm. 10: Microphenocryst (core), the same grain as Analysis No. 9. 11: Phenocryst (core), 472-14-1, 47-53 cm. 12: Phenocryst (rim), the same grain as Analysis No. 11, 13: Microphenocryst (core), 472-14-1, 76-81 cm. 14: Microphenocryst (rim), the same grain as Analysis No. 13. 15: Microphenocryst (rim), 473-29-1, 70-75 cm. 16: Microphenocryst (core), the same grain as
Analysis No. 15. 17: Microphenocryst (core), 473-29-1, 70-75 cm. 18: (core), 473-33-3, 92-97 cm. 19: (core), 473-33-3, 92-97 cm. 20: Phenocryst (core), 473-34-3, 17-23 cm. 21: Phenocryst (rim), the same grain as Analysis No. 


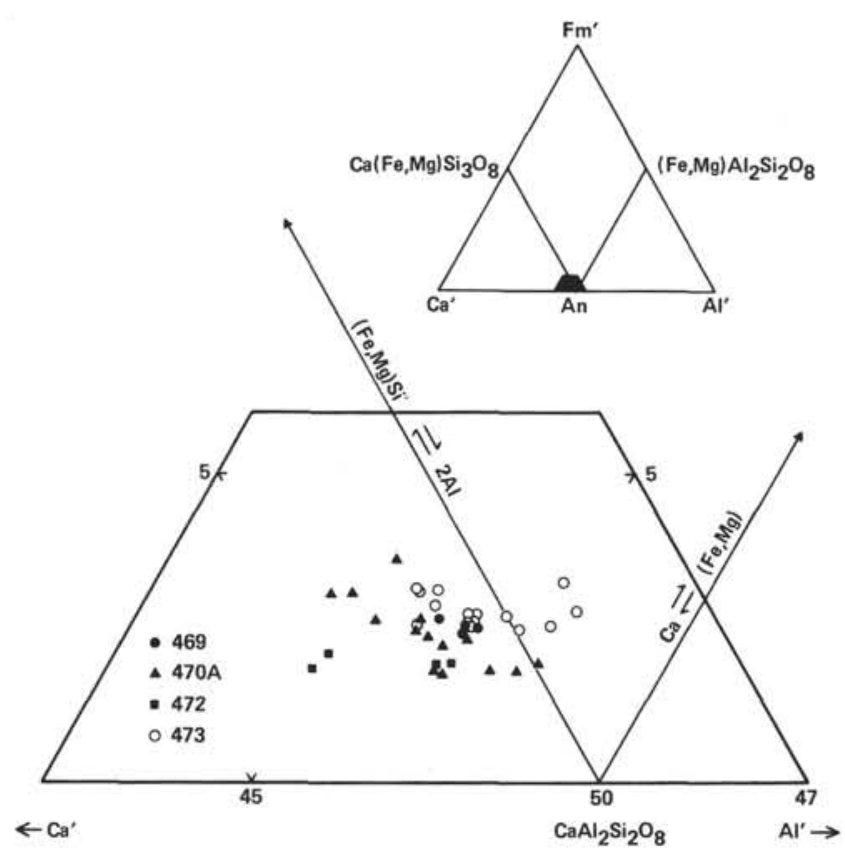

Figure 4. Plagioclase compositions in the plane $\mathrm{Fm}^{\prime}\left[\left(\mathrm{Fe}, \mathrm{Mg}_{2} \mathrm{Si}_{3} \mathrm{O}_{8}\right]\right.$ - $\mathrm{Ca}^{\prime}\left(\mathrm{Ca}_{2}\left(\mathrm{Ca}_{2} \mathrm{Si}_{3} \mathrm{O}_{8}\right)-\mathrm{Al}^{\prime}\left(\mathrm{Al}_{4} \mathrm{SiO}_{8}\right)\right.$ (Longhi, 1976; Longhi et al., 1976).

Microprobe analyses of the fused glass beads and also the natural basalt glasses were obtained with a JEOL X-ray microprobe analyzer at Hiroshima University. The experimental conditions and correction procedures were the same as those described earlier for the mineral analyses except that the glass analyses were obtained with broader electron beam diameters $(\simeq 20 \mu \mathrm{m})$. Analytical precision and data by this method for the USGS reference standard W-1 are given in Table 5. Ferrous iron was determined by titration and $\mathrm{H}_{2} \mathrm{O}$ gravimetrically. $\mathrm{H}_{2} \mathrm{O}^{+}$represents the ignition loss. Trace element analyses were made with the X-ray fluorescence spectrometer at Woods Hole Oceanographic Institution, and the precision and accuracy are of the order $\pm 5 \%$ (Schroeder et al., 1980).

Major and trace element analyses and CIPW normative compositions for 13 Leg 63 basalts are listed in Tables 6 and 7, and petrographic descriptions of these analyzed samples are briefly summarized in Table 8. In addition, microprobe analyses were carried out for 21 Leg 63 natural basalt glasses, and those results are listed in Table 9.

\section{Major and Trace Element Geochemistry}

Relatively extreme iron-enrichment trends have been noted in the differentiation of abyssal tholeiitic magmas from the East Pacific Rise, Juan de Fuca Ridge, and Galapagos spreading center (e.g., Kay et al., 1970; Clague and Bunch, 1976; Schilling et al., 1976), which is one of the characteristics of these rocks compared to their Atlantic counterparts. These tholeiitic basalts define elongated fields parallel to the $\mathrm{FeO} * / \mathrm{MgO}$ side of the AFM diagram as exemplified by the lines GS and SQ in Figure 5. The AFM plot indicates that the Leg 63 basalts and basalt glasses as a whole also define an iron-enrichment trend similar to those just mentioned, but the degree of iron-enrichment is not so extensive. FeO*/ $\mathrm{MgO}$ variation in the Leg 63 basalts of a single drill hole is small; as a result, the distribution of basalts from each drill hole is confined to a small field in the AFM diagram. Collectively, the $\mathrm{FeO}^{*} / \mathrm{MgO}$ ratios of the $\mathrm{Leg}$
Table 5. Analysis of USGS standard rock W-1.

\begin{tabular}{lrr}
\hline Analysis $^{\mathrm{a}}$ & \multicolumn{1}{c}{$\mathrm{A}$} & \multicolumn{1}{c}{$\mathrm{B}$} \\
\hline $\mathrm{SiO}_{2}$ & 53.01 & $52.89 \pm 0.14$ \\
$\mathrm{TiO}_{2}$ & 1.08 & $1.20 \pm 0.01$ \\
$\mathrm{Al}_{2} \mathrm{O}_{3}$ & 15.10 & $15.23 \pm 0.08$ \\
$\mathrm{FeO}^{*}$ & 10.05 & $10.22 \pm 0.04$ \\
$\mathrm{MgO}$ & 6.67 & $6.58 \pm 0.01$ \\
$\mathrm{MnO}$ & 0.17 & $0.19 \pm 0.01$ \\
$\mathrm{CaO}$ & 11.04 & $11.05 \pm 0.11$ \\
$\mathrm{Na} 2$ & 2.16 & $2.10 \pm 0.04$ \\
$\mathrm{~K}_{2} \mathrm{O}$ & 0.64 & $0.62 \pm 0.03$ \\
$\mathrm{P}_{2} \mathrm{O}_{5}$ & 0.14 & \\
$\mathrm{CO}_{2}$ & 0.06 & \\
$\mathrm{Total}$ & 100.12 & \multicolumn{1}{l}{100.08} \\
\hline
\end{tabular}

Note: $\mathrm{FeO}^{*}=$ total iron as $\mathrm{FeO}$.

a Analysis designation: A: From Flanagan (1973), recalculated on the anhydrous basis. B: Mean and standard deviation for triplicate analyses of W-1 glass bead by electron microprobe analysis (Suzuki et al., 1977).

63 basalts and basalt glasses from each drill hole increase in the following order: (1) Hole 470A and 472, (2) Hole 473, and (3) Hole 469.

Although we do not have both bulk rock and glass analyses for the same specimens, the Hole 469 bulk rocks have significantly higher $\mathrm{K}_{2} \mathrm{O}$ content than do the Hole 469 glasses, and the Hole $470 \mathrm{~A}$ bulk rocks tend to have lower $\mathrm{FeO}^{*} / \mathrm{MgO}$ ratios than the Hole $470 \mathrm{~A}$ glasses. Petrographic study indicates that the Hole 469 basalts including the analyzed specimens are intensively altered with the development of smectites in the groundmass. This alteration is also reflected in the relatively higher $\mathrm{Fe}_{2} \mathrm{O}_{3} / \mathrm{FeO}$ and higher $\mathrm{H}_{2} \mathrm{O}$ contents of these basalts (Table 6); apparently the potassium enrichment in the Hole 469 bulk rock relative to the fresh glasses is mainly due to alteration.

In the normative diopside (Di)-olivine (Ol)-quartz (Qz) ternary diagram (Fig. 6), all of the Leg 63 samples with a few exceptions lie on or close to the olivineplagioclase-clinopyroxene cotectic at 1 atm under anhydrous conditions (Walker et al., 1979; Shibata, 1976). Apparently, like many abyssal tholeiites, those samples have reached or are close to three-phase saturation at low pressures. The distribution of the Leg 63 samples in this ternary plot follows the densest cluster of the abyssal tholeiite population (cf. fig. 7 of Walker et al., 1979), indicating that these samples have normative proportions typical of abyssal tholeiites. As with degree of fractionation inferred from the AFM plot, the Leg 63 samples from each drill hole are collectively plotted toward the $\mathrm{Qz}$ apex of the $\mathrm{Di}-\mathrm{Ol}-\mathrm{Qz}$ diagram approximately in the following order: (1) Holes 470A and 472, (2) Hole 473, and (3) Hole 469.

The AFM and normative Di-Ol-Qz plots of the Leg 63 basalt samples indicate that the samples from Holes $470 \mathrm{~A}$ and 472 are less fractionated compared to those from Holes 469 and 473, in accord with their petrography and mineral chemistry described in the previous 
Table 6. Major element compositions and norms of DSDP Leg 63 basalts.

\begin{tabular}{|c|c|c|c|c|c|c|c|c|c|c|c|c|c|}
\hline $\begin{array}{c}\text { Sample } \\
\text { (interval in } \mathrm{cm} \text { ) }\end{array}$ & $\begin{array}{c}469-45-2 \\
24-29\end{array}$ & $\begin{array}{c}469-47-1 \\
63-69\end{array}$ & $\begin{array}{c}470 \mathrm{~A}-7-3 \\
42-50\end{array}$ & $\begin{array}{c}470 \mathrm{~A}-9-1 \\
69-74\end{array}$ & $\begin{array}{c}\text { 470A-9-3, } \\
99-104\end{array}$ & $\begin{array}{c}472-16-1 \\
92-98\end{array}$ & $\begin{array}{c}472 \mathrm{~A}-1-1 \\
0-8\end{array}$ & $\begin{array}{c}473-29-1 \\
70-75\end{array}$ & $\begin{array}{l}473-30-2 \\
114-120\end{array}$ & $\begin{array}{l}473-32-1 \\
108-114\end{array}$ & $\begin{array}{l}473-33-1 \\
115-121\end{array}$ & $\begin{array}{c}473-34-2 \\
51-57\end{array}$ & $\begin{array}{c}473-34-3, \\
26-34\end{array}$ \\
\hline $\mathrm{SiO}_{2}$ & 47.68 & 47.02 & 48.15 & 48.46 & 48.38 & 49.18 & 48.54 & 50.14 & 49.62 & 49.10 & 49.75 & 49.03 & 48.29 \\
\hline $\mathrm{TiO}_{2}$ & 2.06 & 2.28 & 1.21 & 1.37 & 1.36 & 1.74 & 1.69 & 1.70 & 1.60 & 1.51 & 2.01 & 2.00 & 2.30 \\
\hline $\mathrm{Al}_{2} \mathrm{O}_{3}$ & 14.04 & 13.97 & 15.73 & 15.63 & 16.27 & 14.98 & 14.51 & 16.31 & 15.28 & 14.38 & 13.26 & 13.42 & 14.03 \\
\hline $\mathrm{Fe}_{2} \mathrm{O}_{3}$ & 5.90 & 5.98 & 3.30 & 4.47 & 2.84 & 2.29 & 2.88 & 3.54 & 3.39 & 3.11 & 3.30 & 3.30 & 4.10 \\
\hline $\mathrm{FeO}$ & 5.89 & 6.34 & 5.51 & 4.95 & 5.53 & 7.11 & 6.35 & 4.73 & 6.08 & 6.75 & 8.63 & 8.38 & 7.79 \\
\hline $\mathrm{FeO}^{*}$ & 11.20 & 11.72 & 8.48 & 8.97 & 8.09 & 9.17 & 8.94 & 7.92 & 9.13 & 9.55 & 11.60 & 11.35 & 11.48 \\
\hline $\mathrm{MnO}$ & 0.28 & 0.18 & 0.19 & 0.22 & 0.22 & 0.18 & 0.19 & 0.12 & 0.19 & 0.18 & 0.24 & 0.22 & 0.25 \\
\hline $\mathrm{MgO}$ & 6.65 & 6.23 & 9.77 & 7.56 & 7.18 & 7.22 & 6.96 & 6.62 & 7.31 & 7.27 & 6.77 & 7.21 & 6.51 \\
\hline $\mathrm{CaO}$ & 10.16 & 9.04 & 12.00 & 12.19 & 12.41 & 11.53 & 11.33 & 10.12 & 11.81 & 12.18 & 10.99 & 11.08 & 10.79 \\
\hline $\mathrm{Na}_{2} \mathrm{O}$ & 2.61 & 2.96 & 2.05 & 2.60 & 2.61 & 2.51 & 2.34 & 3.15 & 2.73 & 2.78 & 2.76 & 2.66 & 2.91 \\
\hline $\mathrm{K}_{2} \overline{\mathrm{O}}$ & 0.57 & 0.43 & 0.36 & 0.22 & 0.09 & 0.43 & 0.42 & 0.11 & 0.07 & 0.09 & 0.18 & 0.17 & 0.15 \\
\hline $\mathrm{H}_{2} \mathrm{O}^{-}$ & 2.05 & 2.73 & 0.79 & 0.99 & 1.07 & 0.88 & 1.28 & 1.37 & 1.04 & 0.83 & 0.75 & 0.74 & 1.46 \\
\hline $\mathrm{H}_{2} \mathrm{O}^{+}$ & 1.65 & 2.51 & 1.42 & 1.49 & 1.56 & 1.33 & 2.73 & 2.80 & 1.11 & 1.16 & 0.95 & 1.22 & 1.20 \\
\hline Total & 99.54 & 99.67 & 100.48 & 100.15 & 99.52 & 99.38 & 99.22 & 100.71 & 100.23 & 99.34 & 99.59 & 99.43 & 99.78 \\
\hline $\mathrm{FeO} * / \mathrm{MgO}$ & 1.68 & 1.88 & 0.87 & 1.19 & 1.13 & 1.27 & 1.29 & 1.20 & 1.25 & 1.31 & 1.71 & 1.57 & 1.76 \\
\hline \multicolumn{14}{|l|}{ CIPW Norms ${ }^{a}$} \\
\hline Q & 0 & 0 & 0 & 0 & 0 & 0 & 0.49 & 0.58 & 0 & 0 & 0.40 & 0 & 0 \\
\hline or & 3.37 & 2.54 & 2.13 & 1.30 & 0.53 & 2.54 & 2.48 & 0.65 & 0.41 & 0.53 & 1.06 & 1.00 & 0.89 \\
\hline$a b$ & 22.09 & 25.05 & 17.35 & 22.00 & 22.09 & 21.24 & 19.80 & 26.65 & 23.10 & 23.52 & 23.35 & 22.51 & 24.62 \\
\hline an & 24.91 & 23.56 & 32.66 & 30.33 & 32.41 & 28.34 & 27.85 & 30.04 & 29.23 & 26.49 & 23.26 & 24.18 & 24.78 \\
\hline ol & 3.62 & 3.95 & 10.37 & 7.88 & 5.81 & 1.86 & 0 & 0 & 2.33 & 4.86 & 0 & 1.33 & 3.44 \\
\hline hy & 13.40 & 13.71 & 9.18 & 6.19 & 7.36 & 13.72 & 15.62 & 16.54 & 13.29 & 8.54 & 16.86 & 16.04 & 11.91 \\
\hline di & 20.87 & 17.47 & 21.63 & 24.46 & 23.60 & 23.42 & 23.02 & 16.31 & 23.82 & 27.59 & 25.62 & 25.16 & 23.55 \\
\hline $\mathrm{mt}$ & 3.31 & 3.47 & 2.51 & 2.66 & 2.39 & 2.71 & 2.65 & 2.34 & 2.70 & 2.83 & 3.43 & 3.36 & 3.40 \\
\hline il & 3.91 & 4.33 & 2.30 & 2.60 & 2.58 & 3.30 & 3.21 & 3.23 & 3.04 & 2.87 & 3.82 & 3.80 & 4.37 \\
\hline
\end{tabular}

Note: $\mathrm{FeO}^{*}=$ total iron as $\mathrm{FeO}$.

a The norms were calculated after $\mathrm{Fe}_{2} \mathrm{O}_{3}$ was reduced to $\mathrm{FeO}$ so as to have $\mathrm{Fe}_{2} \mathrm{O}_{3} / \mathrm{FeO}=0.25$.

Table 7. Trace element abundances ${ }^{\mathrm{a}}$ of DSDP Leg 63 basalts.

\begin{tabular}{|c|c|c|c|c|c|c|c|c|c|c|c|c|c|}
\hline $\begin{array}{c}\text { Sample } \\
\text { (interval in cm) }\end{array}$ & $\begin{array}{c}469-45-2 \\
24-29\end{array}$ & $\begin{array}{c}469-47-1 \\
63-69\end{array}$ & $\begin{array}{c}470 \mathrm{~A}-7-3 \\
42-50\end{array}$ & $\begin{array}{c}470 \mathrm{~A}-9-1 \\
69-74\end{array}$ & $\begin{array}{c}470 \mathrm{~A}-9-3 \\
99-104\end{array}$ & $\begin{array}{c}472-16-1 \\
92-98\end{array}$ & $\begin{array}{c}472 \mathrm{~A}-1-1 \\
0-8\end{array}$ & $\begin{array}{c}473-29-1 \\
70-75\end{array}$ & $\begin{array}{l}473-30-2 \\
114-120\end{array}$ & $\begin{array}{l}473-32-1 \\
108-114\end{array}$ & $\begin{array}{l}473-33-1 \\
115-121\end{array}$ & $\begin{array}{c}473-34-2 \\
51-57\end{array}$ & $\begin{array}{c}473-34-3, \\
26-34\end{array}$ \\
\hline $\mathrm{Rb}$ & 11.5 & 5.4 & 7.0 & 4.3 & 2.9 & 6.4 & 6.1 & 2.6 & 2.6 & 2.9 & 2.4 & 3.2 & 1.9 \\
\hline $\mathrm{Sr}$ & 80.5 & 80.5 & 133 & 76.2 & 85.6 & 193 & 194 & 127 & 120 & 118 & 103 & 99.8 & 103 \\
\hline $\mathrm{Y}$ & 36.7 & 41.8 & 22.8 & 27.8 & 29.5 & 27.9 & 27.7 & 24.8 & 30.7 & 30.5 & 41.7 & 39.9 & 42.7 \\
\hline $\mathrm{Zr}$ & 95.0 & 108 & 74.2 & 64.3 & 69.0 & 120 & 118 & 97.7 & 90.2 & 89.8 & 118 & 113 & 125 \\
\hline $\mathrm{Nb}$ & $<5$ & $<5$ & 5.2 & $<5$ & $<5$ & 15.3 & 12.7 & $<5$ & $<5$ & $<5$ & $<5$ & 6.0 & $<5$ \\
\hline V & 374 & 423 & 241 & 248 & 247 & 251 & 251 & 314 & 282 & 274 & 353 & 345 & 396 \\
\hline $\mathrm{Cr}$ & 104 & 69.0 & 370 & 283 & 277 & 223 & 227 & 213 & 198 & 194 & 82.9 & 94.0 & 152 \\
\hline Co & 56.6 & 84.1 & 93.2 & 73.5 & 70.9 & 91.2 & 85.6 & 65.4 & 68.6 & 78.6 & 74.2 & 73.5 & 77.7 \\
\hline $\mathrm{Ni}$ & 50.9 & 37.4 & 187 & 91.7 & 99.0 & 85.2 & 82.5 & 61.5 & 56.9 & 57.6 & 46.2 & 57.0 & 51.0 \\
\hline $\mathrm{Cu}$ & 38.7 & 44.7 & 46.6 & 59.1 & 65.9 & 48.2 & 53.4 & 59.4 & 54.0 & 57.4 & 43.3 & 45.3 & 39.2 \\
\hline $\mathrm{Zn}$ & 75.5 & 89.5 & 55.1 & 57.5 & 58.1 & 64.1 & 65.4 & 70.2 & 63.9 & 66.4 & 76.8 & 77.9 & 88.3 \\
\hline
\end{tabular}

a Abundances in ppm.

sections. These compositional characteristics for each basalt group from the Leg 63 drill holes also extend to differences in major and trace element concentrations. For example, the concentrations of $\mathrm{TiO}_{2}, \mathrm{Y}, \mathrm{V}$, and $\mathrm{Zn}$ are enriched, and those of $\mathrm{Al}_{2} \mathrm{O}_{3}, \mathrm{CaO}, \mathrm{Cr}, \mathrm{Ni}$, and $\mathrm{Cu}$ are depleted, in the samples from Holes 469 and 473 relative to those from Holes $470 \mathrm{~A}$ and 472 (Tables 6, 7, and 9).

As exemplified in the $\mathrm{TiO}_{2}$ variation diagram (Fig. 7), oxide and element variations relative to $\mathrm{FeO} * \mathrm{MgO}$ are in most cases systematic, and the Leg 63 samples as a whole appear to define a smooth, continuous trend that we might regard as a liquid line of descent, if the samples were derived from a common parent magma. Such simple compositional variation as observed in the $\mathrm{TiO}_{2}$ diagram, however, is not found in the variation diagrams of $\mathrm{K}_{2} \mathrm{O}, \mathrm{Sr}$, and $\mathrm{Zr}$ (Fig. 8). And it appears that the Hole 472 whole-rock samples at least have $\mathrm{K}_{2} \mathrm{O}$, $\mathrm{Sr}$, and $\mathrm{Zr}$ abundances distinct from other Leg 63 samples.

\section{Oxide Variations of Hole 473}

In the petrography section, we suggested that the massive basalt unit of Hole 473 may represent submarine sheet flows or ponded lava flows. To test compositional variations within the massive basalt unit of Hole 473, we have plotted in Figure 9 the oxide concentrations of the Hole 473 basalts against the depth at which these samples were recovered. This figure indicates that the Hole 473 basalts are chemically more differentiated downward and that, although the number of analyses is small, there appears to be a compositional discontinuity within the massive basalt unit at some horizon in Core 32. This discontinuous horizon, if real, may correspond with the intercalated soft materials that were hit during the drilling of Core 32 . On the basis of this compositional observation, we tentatively divide the basaltic basement at Site 473 into three units (or sheets) with one boundary somewhere in Core 32 and the other in Core 34. 
Table 8. Brief petrographic descriptions of analyzed samples.

\begin{tabular}{|c|c|c|c|c|c|c|}
\hline \multirow{3}{*}{$\begin{array}{c}\text { Sample } \\
\text { (interval in cm) }\end{array}$} & \multirow[b]{3}{*}{ Description } & \multicolumn{5}{|c|}{ Modal Compositions (\%) } \\
\hline & & \multicolumn{4}{|c|}{$\begin{array}{l}\text { Phenocrysts \& } \\
\text { Microphenocrysts }\end{array}$} & \multirow[b]{2}{*}{ Groundmass } \\
\hline & & OI & $\mathrm{Pl}$ & Cpx & $\mathrm{Sp}$ & \\
\hline $469-45-2,24-29$ & $\begin{array}{l}\text { Fine-grained, aphyric, intersertal; acicular plagioclase, } \\
\text { equant clinopyroxene, and opaque minerals; greenish } \\
\text { yellow smectite fills vesicles and replaces interstitial } \\
\text { areas (originally glass?) }\end{array}$ & & & & & \\
\hline $469-47-1,63-69$ & $\begin{array}{l}\text { Very fine-grained, very rare plagioclase micropheno- } \\
\text { crysts, variolitic; acicular plagioclase, clinopyroxene, } \\
\text { and opaque mineral; yellowish brown smectite fills } \\
\text { vesicles }\end{array}$ & $-^{a}$ & tr. & - & - & 100.0 \\
\hline $470 \mathrm{~A}-7-3,42-50$ & $\begin{array}{l}\text { Fine grained, porphyritic; intersertal groundmass of } \\
\text { acicular plagioclase, feathery clinopyroxene, equant } \\
\text { brown spinel, and irregular, minute opaque mineral; } \\
\text { yellowish brown smectite pseudomorphs of euhedral, } \\
\text { olivine phenocrysts }\end{array}$ & 2.5 & 1.8 & - & tr. & 95.7 \\
\hline 470A-9-1, 69-74 & $\begin{array}{l}\text { Fine-grained, glomeroporphyritic with variolitic } \\
\text { groundmass; acicular, skeletal plagioclase, feathery } \\
\text { clinopyroxene, equant olivine, and irregular, minute } \\
\text { opaque mineral }\end{array}$ & - & 0.3 & - & - & 99.7 \\
\hline $470 \mathrm{~A}-9-3,99-104$ & $\begin{array}{l}\text { Medium-grained, aphyric, intersertal to subophitic; } \\
\text { lathy plagioclase, subhedral clinopyroxene, small } \\
\text { granules of olivine, and interstitial, irregular, opaque } \\
\text { mineral; brown smectite partly or completely replaces } \\
\text { olivine }\end{array}$ & & & & & \\
\hline $472-16-1,92-98$ & $\begin{array}{l}\text { Fine- to medium-grained, porphyritic, subvariolitic } \\
\text { groundmass of acicular, skeletal plagioclase, feathery, } \\
\text { dendritic clinopyroxene, and minute opaque minerals; } \\
\text { brown smectite fills vesicles and replaces olivine } \\
\text { microphenocrysts }\end{array}$ & 0.3 & 1.5 & 0.2 & - & 98.0 \\
\hline $472 A-1-1,0-8$ & $\begin{array}{l}\text { Essentially similar to Sample } 472-16-1,92-98 \text {, except } \\
\text { for no pyroxene microphenocryst }\end{array}$ & 0.9 & 1.7 & - & - & 97.4 \\
\hline $473-29-1,70-75$ & $\begin{array}{l}\text { Fine-grained, glomeroporphyritic; intergranular to } \\
\text { intersertal groundmass of plagioclase laths, } \\
\text { clinopyroxene granules, and minute opaque grains; } \\
\text { smectite pseudomorphing olivine microphenocrysts }\end{array}$ & 1.0 & 5.6 & 0.4 & - & 93.0 \\
\hline $473-30-2,114-120$ & $\begin{array}{l}\text { Medium-grained, aphyric, intersertal to subophitic; } \\
\text { subhedral plagioclase, anhedral clinopyroxene, and } \\
\text { anhedral opaque mineral; clay-rich altered interstices }\end{array}$ & & & & & \\
\hline $473-32-1,108-114$ & Essentially similar to Sample $473-30-2,114-120$ & & & & & \\
\hline $473-33-1,115-121$ & Essentially similar to Sample $473-30-2,114-120$ & & & & & \\
\hline $473-34-2,51-57$ & Essentially similar to Sample $473-30-2,114-120$ & & & & & \\
\hline $473-34-3,26-34$ & $\begin{array}{l}\text { Fine-grained, glomeroporphyritic with hyalo-ophitic } \\
\text { groundmass; skeletal, hollow groundmass plagioclase, } \\
\text { feathery groundmass clinopyroxene }\end{array}$ & - & 5.2 & 2.5 & - & 92.3 \\
\hline
\end{tabular}

Note: $\mathrm{Ol}, \mathrm{Pl}, \mathrm{Cpx}$, and $\mathrm{Sp}$ indicate olivine, plagioclase, clinopyroxene, and spinel, respectively.

$\mathrm{a}_{-}=$not present.

\section{DISCUSSION}

The Leg 63 basalts resemble abyssal tholeiites in their major and trace element chemistry; in particular, they are very similar to those described from the Eastern Pacific ocean floor (Tables 10 and 11). Hole 470A was cored east of Guadalupe Island and $8 \mathrm{~km}$ southsouthwest of the Experimental Mohole site drilled in 1961. Analytical data for the basalt recovered at the Experimental Mohole (EM) site are listed in Table 10 (Engel and Engel, 1961) and are compared with the Hole $470 \mathrm{~A}$ basalts. The Mohole basalt and our Hole $470 \mathrm{~A}$ basalts are very similar to each other; the surface of the oceanic basement east of Guadalupe Island is apparently made up of rather uniform tholeiitic basalts.

Compositional variation in the Leg 63 basalts recovered from any single drill hole is not extensive. However, the Leg 63 samples (except those of Hole 472) define continuous, smooth trends in oxide and element variation diagrams relative to $\mathrm{FeO} * / \mathrm{MgO}$ and lie on or close to the olivine-plagioclase-clinopyroxene cotectic at low pressures in the normative Di-Ol-Qz diagram. These observations may indicate that the samples from Holes $469,470 \mathrm{~A}$, and 473 evolved by shallow-level frac- tional crystallization of similar parental magma(s), although the Leg 63 drill sites where these samples were recovered are located at significant distances from each other.

\section{ACKNOWLEDGMENTS}

We thank A. Minami of Hiroshima University for making the microprobe analyses presented in this report; T. Saito of Okayama University for technical assistance; and J. F. Bender and D. J. Fornari for critical comments. This work was supported by the Japan Society for the Promotion of Science and the U.S. National Science Foundation (Grants OCE76-21882 and INT78-22980).

\section{REFERENCES}

Ballard, R. D., Holcomb, R. T., and van Andel, Tj. H., 1979. The Galapagos rift at $86^{\circ} \mathrm{W}: 3$. sheet flows, collapse pits, and lava lakes of the rift valley. J. Geophys. Res., 84:5407-5422.

Batiza, R., Rosendahl, B. R., and Fisher, R. L., 1977. Evolution of oceanic crust. 3. Petrology and chemistry of basalts from the East pacific rise and the Siqueiros transform fault. J. Geophys. Res., $82: 265-276$.

Bence, A. E., and Albee, A. L., 1968. Empirical correction factors for the electron microanalysis of silicates and oxides. J. Geol., 76: $382-403$.

Bryan, W. B., 1974. Fe-Mg relationships in sector-zoned submarine basalt plagioclase. Earth Plant. Sci. Lett., 24:157-165. 
1979. Regional variation and petrogenesis of basalt glasses from the Famous area, Mid-Atlantic Ridge. J. Petrol., 20:293325.

Bryan, W. B., and Moore, J. G., 1977. Compositional variations of young basalts in the Mid-Atlantic Ridge rift valley near $36^{\circ} 49^{\prime} \mathrm{N}$. Geol. Soc. Am. Bull., 88:556-570.

Clague, D. A., and Bunch, T. E., 1976. Formation of ferrobasalt at East Pacific mid-ocean spreading centers. J. Geophys. Res., 81: 4247-4256.

Engel, A. E. J., Engel, C. G., and Havens, R. G., 1965. Chemical characteristics of oceanic basalts and the upper mantle. Geol. Soc. Am. Bull., 76:719-734.

Engel, C. G., and Engel, A. E. J., 1961. Composition of basalt cored in Mohole project. Am. Assoc. Petrol. Geol. Bull., 45:1799.

Finger, L. W., 1972. The uncertainty in the calculated ferric iron content of microprobe analyses. Year Book-Carnegie Inst. Washington Ann. Rep. Director Geophys. Lab., 71:600-603.

Flanagan, F. J., 1973. 1972 values for international geochemical reference samples. Geochim. Cosmochim. Acta, 37:1189-1201.

Frey, F. A., Bryan, W. B., and Thompson, G., 1974. Atlantic Ocean floor: geochemistry and petrology of basalts from Legs 2 and 3 of the Deep-Sea Drilling Project. J. Geophys. Res., 79:5507-5527.

Hall, J. M., and Robinson, P. T., 1979. Deep crustal drilling in the North Atlantic Ocean. Science, 204:573-586.

Irvine, T. N., 1967. Chromian spinel as a petrogenetic indicator. 2. Petrologic applications. Can. J. Earth Sci., 4:71-103.

Kay, R., Hubbard, N. J., and Gast, P. W., 1970. Chemical characteristics and origin of ocean ridge volcanic rocks. J. Geophys. Res., 75:1586-1613.

Longhi, J., 1976. Iron, magnesium and silica in plagioclase [Ph.D. thesis]. Harvard University.

Longhi, J., Walker, D., and Hays, J. F., 1976. Fe and Mg in plagioclase. Proc. 7th Lunar Sci. Conf., pp. 1281-1300.

Melson, W. G., Vallier, T. L., Wright, T. L., et al., 1976. Chemical diversity of abyssal volcanic glass erupted along Pacific, Atlantic, and Indian ocean sea-floor spreading centers. In Sutton, G. H., et al. (Eds.), The Geophysics of the Pacific Ocean Basin and Its Margin: Geophys. Monogr. 19: Washington (AGU), pp. 351-367.

Morel, J. M., and Hekinian, R., 1980. Compositional variations of volcanics along segments of recent spreading ridges. Contrib. Mineral. Petrol., 72:425-436.
Nakamura, Y., and Kushiro, I., 1970. Compositional relations of coexisting orthopyroxene, pigeonite and augite in a tholeiitic andesite from Hakone volcano. Contrib. Mineral. Petrol., 29:265-275.

Roeder, P. L., and Emslie, R. F., 1970. Olivine-liquid equilibrium. Contrib. Mineral. Petrol., 29:275-289.

Schilling, J.-G., Anderson, R. N., and Vogt, P., 1976. Rare earth, Fe, and $\mathrm{Ti}$ variations along the Galapagos spreading centre, and their relationship to the Galapagos mantle plume. Nature, 261:108-113.

Schroeder, B., Thompson, G., Sulanowski, M., et al., 1980. Analysis of geologic materials using an automated X-ray fluorescence system. X-Ray Spectrom., 9:198-205.

Shibata, T., 1976. Phenocryst-bulk rock composition relations of abyssal tholeiites and their petrogenetic significance. Geochim. Cosmochim. Acta, 40:1407-1417.

Shibata, T., DeLong, S. E., and Walker, D., 1979. Abyssal tholeiites from the Oceanographer Fracture Zone, I. Petrology and fractionation. Contrib. Mineral. Petrol., 70:89-102.

Shibata, T., Thompson, G., and Frey, F. A., 1979. Tholeiitic and alkalai basalts from the Mid-Atlantic Ridge at $43^{\circ} \mathrm{N}$. Contrib. Mineral. Petrol., 70:127-141.

Sigurdsson, H., and Schilling, J.-G., 1976. Spinels in Mid-Atlantic Ridge basalts: chemistry and occurrence. Earth Planet. Sci. Lett., 29:7-20.

Suzuki, M., Minami, A., and Yokoyama, S., 1977. Major element bulk rock analysis using electron microprobe analyzer (in Japanese). Joint. Ann. Mtg. Soc. Min. Geol. Japan, Japan: Assoc. Petrol. Mineral. Econ. Geol., Mineral. Soc. Japan, Abs. with Programs, p. 41.

Thompson, G., Bryan, W. B., Frey, G. A., et al., 1976. Petrology and geochemistry of basalts from DSDP Leg 34, Nazca Plate. In Yeats, R. S., Hart, S. R., et al., Init. Repts. DSDP, 34: Washington (U.S. Govt. Printing Office), 215-225.

Walker, D., Kirkpatrick, R. J., Longhi, J., et al., 1976. Crystallization history of lunar picritic basalt sample 12002: phase-equilibria and cooling-rate studies. Geol. Soc. Am. Bull., 87:646-656.

Walker, D., Shibata, T., and DeLong, S. E., 1979. Abyssal tholeiites from the Oceanographer Fracture Zone, II. Phase equilibria and mixing. Contrib. Mineral. Petrol. 70:111-125.

Table 9. Microprobe analyses of DSDP Leg 63 basalt glasses.

\begin{tabular}{|c|c|c|c|c|c|c|c|c|c|c|}
\hline $\begin{array}{c}\text { Sample } \\
\text { (interval in } \mathrm{cm} \text { ) }\end{array}$ & $\begin{array}{c}469-48-1 \\
43-48\end{array}$ & $\begin{array}{c}469-48-1 \\
54-61\end{array}$ & $\begin{array}{c}469-48-1 \\
98-100\end{array}$ & $\begin{array}{l}469-48-1 \\
102-107\end{array}$ & $\begin{array}{c}469-49-2, \\
16-20\end{array}$ & $\begin{array}{c}469-50-1 \\
57-61\end{array}$ & $\begin{array}{c}469-50-1, \\
89-91\end{array}$ & $\begin{array}{r}469-50-1 \\
113-117\end{array}$ & $\begin{array}{c}469-50-2 \\
20-22\end{array}$ & $\begin{array}{c}470 \mathrm{~A}-8-2 \\
56-60\end{array}$ \\
\hline $\mathrm{SiO}_{2}$ & 49.78 & 49.70 & 50.28 & 50.30 & 50.10 & 50.74 & 50.81 & 50.14 & 50.74 & 50.26 \\
\hline $\mathrm{TiO}_{2}$ & 1.94 & 2.00 & 1.81 & 1.78 & 2.01 & 1.88 & 1.96 & 1.96 & 1.95 & 1.68 \\
\hline $\mathrm{Al}_{2} \mathrm{O}_{3}$ & 13.45 & 13.49 & 13.19 & 14.04 & 13.33 & 13.75 & 13.58 & 13.55 & 12.91 & 14.61 \\
\hline $\mathrm{FeO}^{*}$ & 11.88 & 11.88 & 11.55 & 11.52 & 11.85 & 11.69 & 11.98 & 11.97 & 11.66 & 9.31 \\
\hline $\mathrm{MnO}$ & 0.26 & 0.26 & 0.19 & 0.26 & 0.25 & 0.26 & 0.20 & 0.22 & 0.21 & 0.20 \\
\hline $\mathrm{MgO}$ & 6.88 & 6.86 & 7.13 & 7.48 & 7.17 & 6.42 & 7.71 & 7.26 & 7.09 & 7.17 \\
\hline $\mathrm{CaO}$ & 11.26 & 11.31 & 11.59 & 11.62 & 11.24 & 11.97 & 11.05 & 11.36 & 11.20 & 11.72 \\
\hline $\mathrm{Na}_{2} \mathrm{O}$ & 2.70 & 2.71 & 2.53 & 2.66 & 2.71 & 2.10 & 2.81 & 2.87 & 2.85 & 2.83 \\
\hline $\mathrm{K}_{2} \mathrm{O}$ & 0.10 & 0.11 & 0.09 & 0.10 & 0.11 & 0.10 & 0.10 & 0.12 & 0.12 & 0.52 \\
\hline Total & 98.25 & 98.32 & 98.36 & 99.76 & 98.77 & 98.91 & 100.20 & 99.45 & 98.73 & 98.30 \\
\hline $\mathrm{FeO}^{*} / \mathrm{MgO}$ & 1.73 & 1.73 & 1.62 & 1.54 & 1.65 & 1.82 & 1.55 & 1.65 & 1.65 & 1.30 \\
\hline
\end{tabular}

Note: $\mathrm{FeO}^{*}=$ total iron as $\mathrm{FeO}$. 
Table 9. (Continued).

\begin{tabular}{|c|c|c|c|c|c|c|c|c|c|c|}
\hline $\begin{array}{c}470 \mathrm{~A}-8-3, \\
76-81\end{array}$ & $\begin{array}{c}470 \mathrm{~A}-8-5 \\
74-80\end{array}$ & $\begin{array}{c}470 \mathrm{~A}-10-1, \\
16-21\end{array}$ & $\begin{array}{c}470 \mathrm{~A}-13-1, \\
38-41\end{array}$ & $\begin{array}{c}472-14-1, \\
5-11\end{array}$ & $\begin{array}{c}472-14-1, \\
32-38\end{array}$ & $\begin{array}{c}472-14-1 \\
47-53\end{array}$ & $\begin{array}{c}472-14-1 \\
69-73\end{array}$ & $\begin{array}{c}472-14-1 \\
76-81\end{array}$ & $\begin{array}{c}472-14-2, \\
45-51\end{array}$ & $\begin{array}{c}472-16-1 \\
120-124\end{array}$ \\
\hline 49.77 & 49.66 & 50.03 & 50.26 & 49.29 & 49.89 & 50.66 & 50.43 & 49.62 & 50.48 & 50.41 \\
\hline 1.54 & 1.53 & 1.43 & 1.93 & 1.39 & 1.36 & 1.30 & 1.36 & 1.25 & 1.42 & 1.75 \\
\hline 15.20 & 14.37 & 15.42 & 13.83 & 14.58 & 15.11 & 14.45 & 15.04 & 15.02 & 14.63 & 15.39 \\
\hline 9.30 & 9.35 & 9.26 & 10.67 & 9.14 & 9.11 & 8.42 & 9.11 & 9.04 & 9.40 & 9.24 \\
\hline 0.17 & 0.17 & 0.20 & 0.20 & 0.14 & 0.18 & 0.05 & 0.13 & 0.19 & 0.20 & 0.19 \\
\hline 8.25 & 8.28 & 8.13 & 7.91 & 8.41 & 8.47 & 8.65 & 8.16 & 8.54 & 7.87 & 7.24 \\
\hline 12.60 & 12.58 & 12.33 & 11.49 & 12.64 & 12.63 & 11.99 & 12.40 & 12.34 & 12.71 & 11.53 \\
\hline 2.74 & 2.74 & 2.79 & 2.67 & 2.60 & 2.46 & 2.85 & 2.71 & 2.51 & 2.48 & 2.78 \\
\hline 0.06 & 0.08 & 0.08 & 0.14 & 0.18 & 0.20 & 0.21 & 0.25 & 0.19 & 0.15 & 0.50 \\
\hline 99.63 & 98.76 & 99.67 & 99.10 & 98.37 & 99.41 & 98.58 & 99.59 & 98.70 & 99.34 & 99.03 \\
\hline 1.13 & 1.13 & 1.14 & 1.35 & 1.09 & 1.08 & 0.97 & 1.12 & 1.06 & 1.19 & 1.28 \\
\hline
\end{tabular}

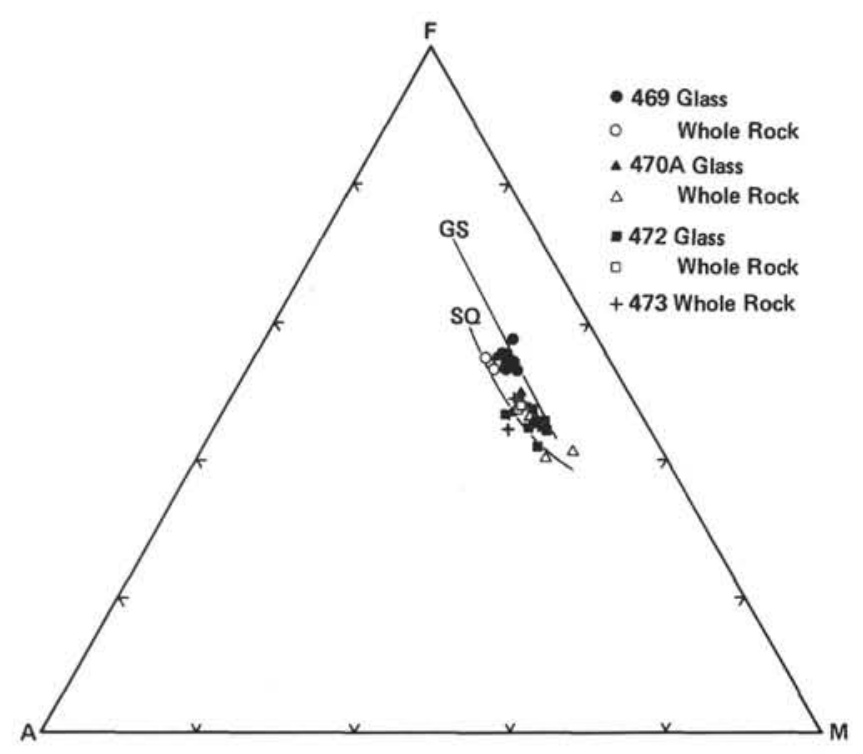

Figure 5. AFM diagram, $\left(\mathrm{Na}_{2} \mathrm{O}+\mathrm{K}_{2} \mathrm{O}\right)-\mathrm{FeO}^{*}-\mathrm{MgO}$, showing the distribution of DSDP Leg 63 tholeiites and basalt glasses. (The lines GS and SQ indicate the fractionation trends of tholeiitic basalts from the Galapagos spreading center [Schilling et al., 1976] and from the Siqueiros fracture zone [Batiza et al., 1977], respectively.)

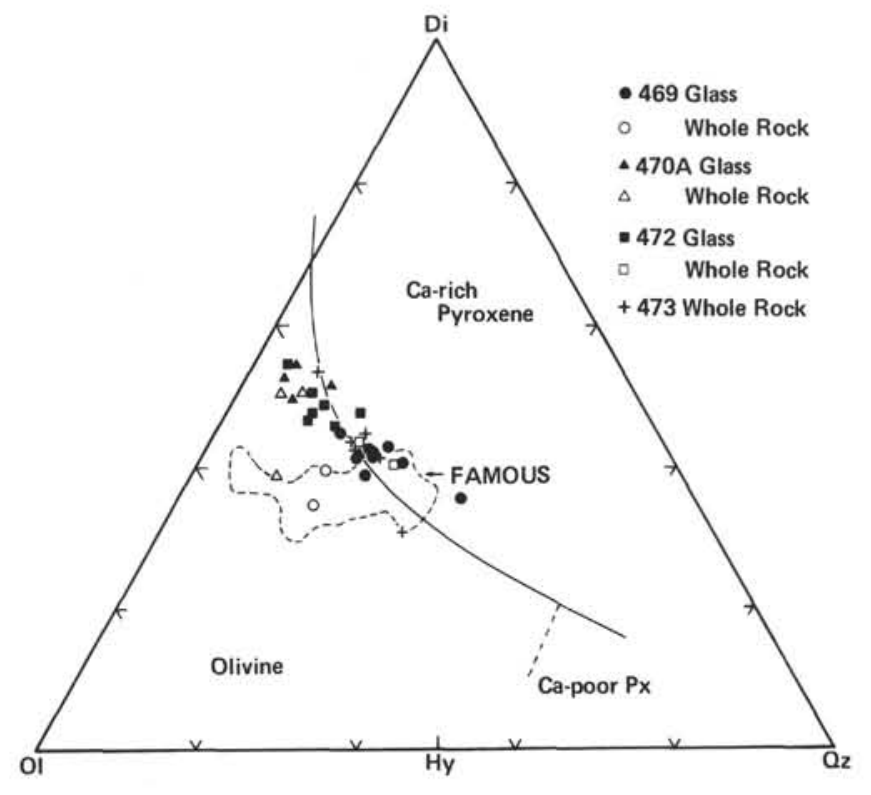

Figure 6. Normative plots of DSDP Leg 63 tholeiites and basalt glasses in molecular proportions; the CIPW norms were calculated on the basis of all iron present as $\mathrm{FeO}$. Ol: $(\mathrm{Mg}, \mathrm{Fe})_{2} \mathrm{SiO}_{4}, \mathrm{Di}$ : $\mathrm{Ca}(\mathrm{Mg}, \mathrm{Fe}) \mathrm{Si}_{2} \mathrm{O}_{6}, \mathrm{Hy}:(\mathrm{Mg}, \mathrm{Fe}) \mathrm{SiO}_{3}, \mathrm{Qz}: \mathrm{SiO}_{2}$. (The solid line is the olivine-plagioclase-clinopyroxene cotectic determined experimentally at 1 atm for the Oceanographer fracture zone basalts [Walker et al., 1979]. The field for the FAMOUS glasses is enclosed with a broken line [Bryan and Moore, 1977; Bryan, 1979].) 


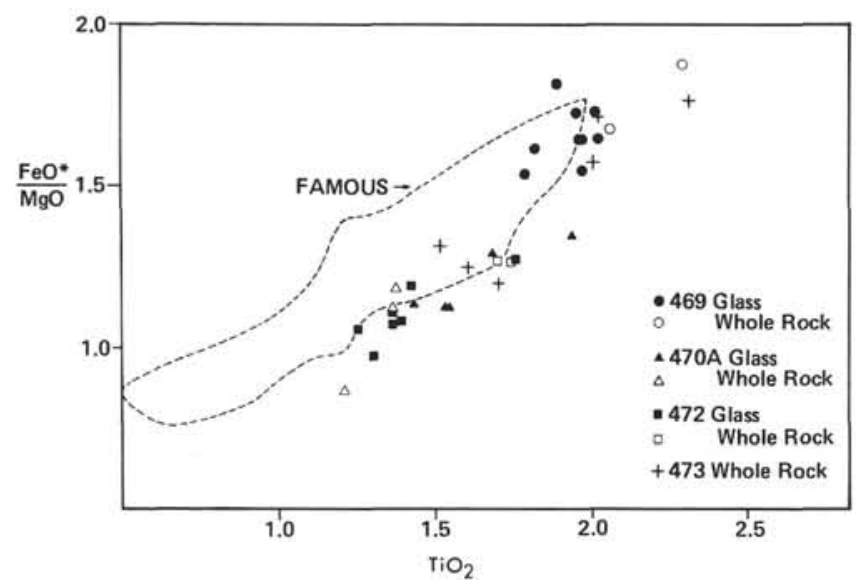

Figure 7. $\mathrm{TiO}_{2}$ abundances versus $\mathrm{FeO}^{*} / \mathrm{MgO}$. (The field for the FAMOUS glasses is enclosed with a broken line [Bryan and Moore, 1977; Bryan, 1979].)

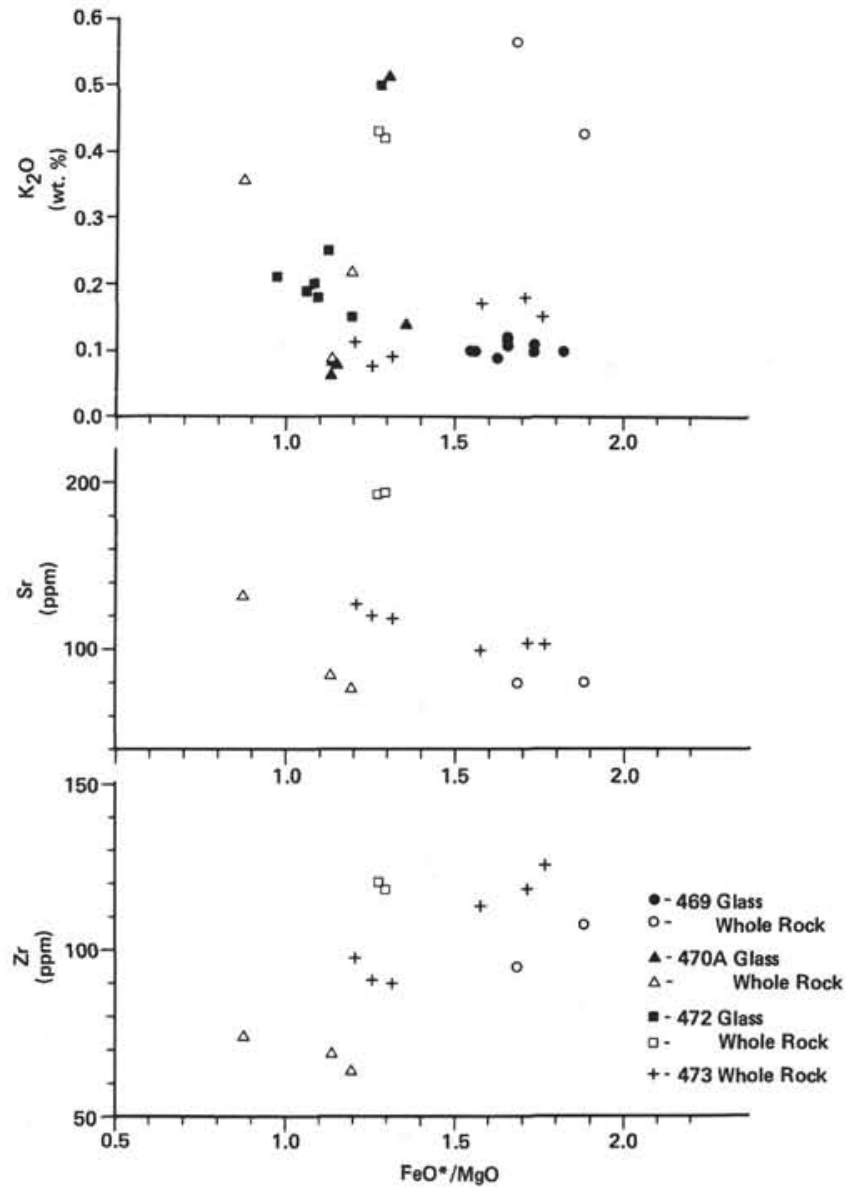

Figure 8. Variations of $\mathrm{K}_{2} \mathrm{O}, \mathrm{Sr}$, and $\mathrm{Zr}$ as a function of $\mathrm{FeO} * / \mathrm{MgO}$. 


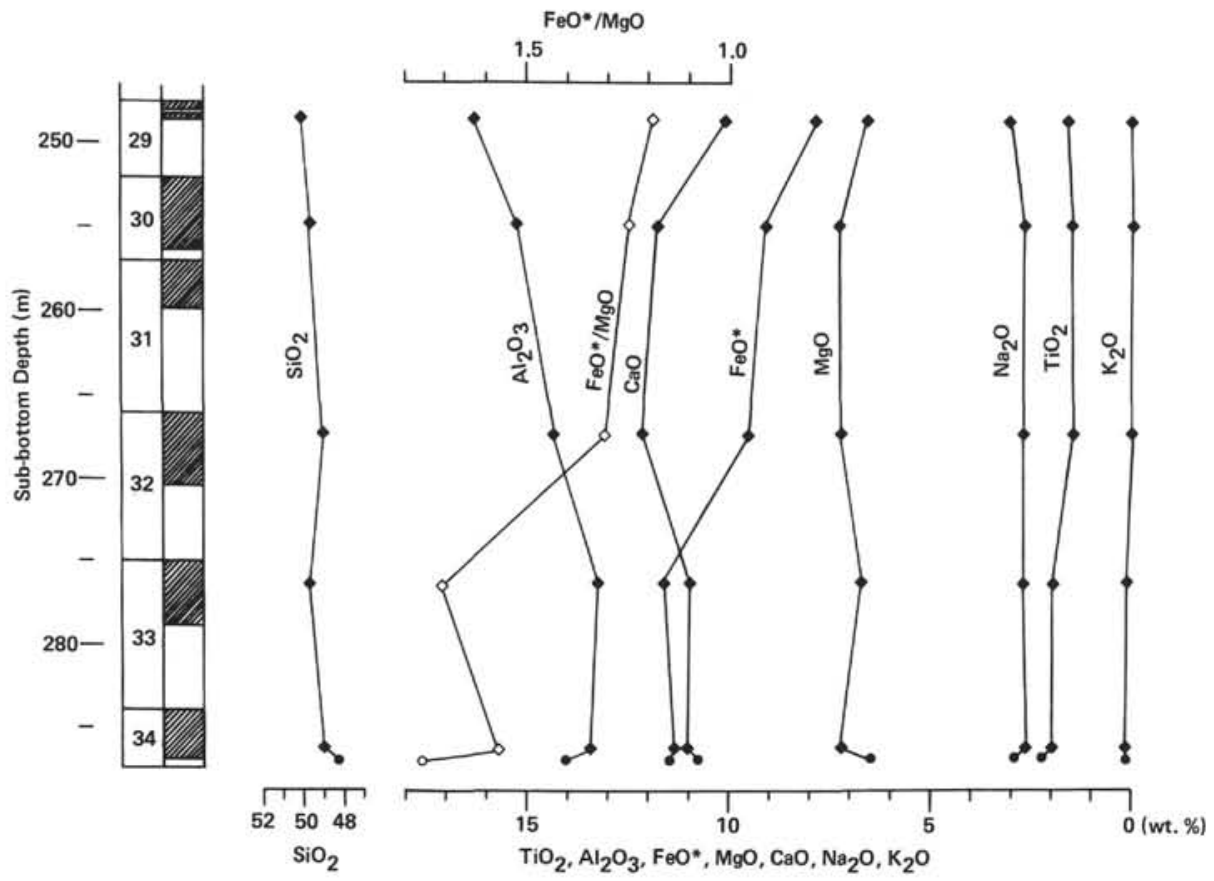

Figure 9. Oxide abundances and $\mathrm{FeO}^{*} / \mathrm{MgO}$ variation versus sub-bottom depth at Site 473, DSDP Leg 63. (Depths are in meters from the sediment/seawater interface.)

Table 10. Comparison of DSDP Leg 63 basalts with other reported East Pacific Rise basalts.

\begin{tabular}{|c|c|c|c|c|c|c|c|c|}
\hline Sample $^{\mathrm{a}}$ & EM & D4 & $\begin{array}{l}\text { Leg } 34 \\
\text { Grp. } 1\end{array}$ & $\begin{array}{l}\text { Leg } 34 \\
\text { Grp. V }\end{array}$ & SD8-3 & SD6-3 & $\begin{array}{c}\text { Leg } 63 \\
470 \mathrm{~A}- \\
7-3\end{array}$ & $\begin{array}{c}\text { Leg } 63 \\
469- \\
47-1\end{array}$ \\
\hline $\mathrm{SiO}_{2}$ & 49.13 & 50.40 & 51.78 & 51.6 & 50.3 & 48.00 & 48.15 & 47.02 \\
\hline $\mathrm{TiO}_{2}$ & 1.23 & 1.60 & 1.17 & 2.4 & 0.93 & 2.70 & 1.21 & 2.28 \\
\hline $\mathrm{Al}_{2} \mathrm{O}_{3}$ & 14.97 & 14.30 & 14.74 & 13.8 & 15.6 & 12.8 & 15.73 & 13.97 \\
\hline $\mathrm{Fe}_{2} \mathrm{O}_{3}$ & 3.28 & - & - & - & 1.27 & 2.22 & 3.30 & 5.98 \\
\hline $\mathrm{FeO}$ & 5.72 & $10.00^{\circ}$ & $9.62^{\circ}$ & $13.1^{\circ}$ & 7.45 & 11.66 & 5.51 & 6.34 \\
\hline $\mathrm{MnO}$ & 0.16 & - & 0.16 & 0.2 & 0.14 & 0.24 & 0.19 & 0.18 \\
\hline $\mathrm{MgO}$ & 7.68 & 7.10 & 8.05 & 6.6 & 9.80 & 5.98 & 9.77 & 6.23 \\
\hline $\mathrm{CaO}$ & 12.68 & 12.30 & 11.82 & 10.3 & 12.77 & 10.14 & 12.00 & 9.04 \\
\hline $\mathrm{Na}_{2} \mathrm{O}$ & 2.37 & 2.78 & 2.46 & 2.5 & 2.41 & 3.33 & 2.05 & 2.96 \\
\hline $\mathrm{K}_{2} \mathrm{O}$ & 0.16 & 0.12 & 0.04 & - & 0.05 & 0.27 & 0.36 & 0.43 \\
\hline $\mathrm{H}_{2} \mathrm{O}^{-}$ & 1.25 & - & - & - & - & - & 0.79 & 2.73 \\
\hline $\mathrm{H}_{2} \mathrm{O}^{+}$ & 1.06 & - & - & - & - & - & 1.42 & 2.51 \\
\hline $\mathrm{P}_{2} \mathrm{O}_{5}$ & 0.15 & - & - & - & 0.09 & 0.23 & - & - \\
\hline Total & 99.84 & 98.60 & 99.84 & 100.5 & 100.81 & 97.57 & 100.48 & 99.67 \\
\hline $\mathrm{FeO} * / \mathrm{MgO}$ & 1.13 & 1.41 & 1.20 & 1,98 & 0.88 & 2.28 & 0.87 & 1.88 \\
\hline $\mathbf{R b}$ & $<10$ & - & - & - & $<5$ & $<5$ & 7.0 & 5.4 \\
\hline $\mathrm{Sr}$ & 97 & 125 & 95 & 82 & 125 & 140 & 133 & 80.5 \\
\hline $\mathbf{Y}$ & 38 & - & 33 & 65 & - & - & 22.8 & 41.8 \\
\hline $\mathrm{Zr}$ & 67 & - & 81 & 178 & - & - & 74.2 & 108 \\
\hline $\mathrm{Nb}$ & $<30$ & - & - & - & - & - & 5.2 & $<5$ \\
\hline v & 320 & - & 261 & 431 & 227 & 397 & 241 & 423 \\
\hline $\mathrm{Cr}$ & 460 & - & 250 & 112 & 490 & 57 & 370 & 69.0 \\
\hline Co & 35 & - & 49 & 44 & 45 & 54 & 93.2 & 84.1 \\
\hline $\mathrm{Ni}$ & 110 & - & 81 & 66 & 130 & 43 & 187 & 37.4 \\
\hline $\mathrm{Cu}$ & 83 & - & 91 & 52 & 82 & 50 & 46.6 & 44.7 \\
\hline $\mathrm{Zn}$ & - & - & - & - & - & - & 55.1 & 89.5 \\
\hline
\end{tabular}

Note: $\cdot=$ total iron as $\mathrm{FeO}$.

A Samples are EM: Mohole basalt (Engel and Engel, 1961; Engel et al, 1965). D4: East Pacific Rise $18^{\circ} 52^{\prime} \mathrm{S}, 113^{\circ} 19^{\prime} \mathrm{W}$ microphyric basalt (Kay et al., 1970). Grp. I, Grp. V: DSDP Leg

34 basalts from the Nazca Plate (Thompson et al., 1976). SD8-3, SD6-3: From the Siqueiros

fracture zone (Batiza et al., 1977).
Table 11. Comparison of average Leg 63 basalt glasses with other reported average ocean ridge basalt glass analyses.

\begin{tabular}{|c|c|c|c|c|c|}
\hline \multirow[b]{2}{*}{ Sample ${ }^{a}$} & \multirow[b]{2}{*}{ EPR } & \multirow[b]{2}{*}{ JFR } & \multicolumn{3}{|c|}{ Leg 63} \\
\hline & & & Site 469 & Hole 470A & Site 472 \\
\hline $\mathrm{SiO}_{2}$ & $49.96 \pm 0.80$ & 50.06 & 50.29 & 50.00 & 50.11 \\
\hline $\mathrm{TiO}_{2}$ & $1.72 \pm 0.48$ & 1.95 & 1.92 & 1.62 & 1.40 \\
\hline $\mathrm{Al}_{2} \mathrm{O}_{3}$ & $15.02 \pm 1.22$ & 14.76 & 13.48 & 14.69 & 14.89 \\
\hline $\mathrm{FeO}^{*}$ & $11.04 \pm 1.45$ & 12.03 & 11.78 & 9.58 & 9.07 \\
\hline $\mathrm{MnO}$ & - & - & 0.23 & 0.19 & 0.15 \\
\hline $\mathrm{MgO}$ & $7.23 \pm 1.04$ & 6.81 & 7.11 & 7.95 & 8.19 \\
\hline $\mathrm{CaO}$ & $11.23 \pm 1.17$ & 11.12 & 11.40 & 12.14 & 12.32 \\
\hline $\mathrm{Na}_{2} \mathrm{O}$ & $2.76 \pm 0.25$ & 2.72 & 2.66 & 2.75 & 2.63 \\
\hline $\mathrm{K}_{2} \mathrm{O}$ & $0.17 \pm 0.14$ & 0.21 & 0.11 & 0.18 & 0.24 \\
\hline $\mathrm{P}_{2} \mathrm{O}_{5}$ & $0.15 \pm 0.08$ & 0.16 & - & - & - \\
\hline Total & 99.30 & 99.82 & 98.98 & 99.10 & 99.00 \\
\hline
\end{tabular}

a Samples are: EPR: Average of 89 basaltic glass analyses from the East Pacific Rise (Morel and Hekinian, 1980). JFR: Average of 18 basaltic glass analyses from the Juan de Fuca Ridge (Melson et al., 1976). 